New Concepts in Imaging: Optical and Statistical Models

D. Mary, C. Theys and C. Aime (eds)

EAS Publications Series, 59 (2013) 93-130

\title{
ADAPTIVE OPTICS FEEDBACK CONTROL
}

\author{
J.-P. Folcher ${ }^{1}$, M. Carbillet $^{1}$, A. Ferrari ${ }^{1}$ and A. Abelli ${ }^{1}$
}

\begin{abstract}
This paper concentrates on the control aspects of Adaptive Optics (AO) systems and includes a prior exposure to linear control systems from the "classical" point of view. The AO control problem is presented and the well-established optimized modal gain integral control approach is discussed. The design of a controller from a modern control point of view is addressed by means of a linear quadratic Gaussian control methodology. The proposed approach emphasizes the ability of the adaptive optics loop to reject the atmospheric aberration. We derive a diagonal state space system which clearly separates the dynamics of the plant (deformable mirror \& wavefront sensor) from the disturbance dynamics (atmospheric model). This representation facilitates the numerical resolution of the problem. A frequency analysis is carried out to check performance and robustness specifications of the multiple-input multiple-output feedback system. The effectiveness of the approach is demonstrated through numerical experiments.
\end{abstract}

\section{Introduction}

Among its applications, adaptive optics systems can be used to reduce the effects of atmospheric turbulence on images taken from ground-based telescopes. A Deformable Mirror (DM) is used to spatially compensate the incoming (atmospheric) wavefront as close as possible to a theoretical plane wavefront. The shape of the DM is adjusted in real time using the measurements of a Wavefront Sensor (WFS) which provides the local slopes of the residual wavefront. The AO system imaging performance depends mainly on the WFS and DM characteristics and on the control algorithm efficiency. For an overview of AO, the reader may consult the book of Roddier (1999) and the companion chapter of Carbillet in this book. This paper concentrates on the control aspects of AO systems. Our intended audience includes researchers and research students in astrophysics and in signal processing

1 UMR 7293, Lagrange Université de Nice Sophia-Antipolis/CNRS/Observatoire de la Côte d'Azur, Parc Valrose, 06108 Nice Cedex 2, France 
who are not familiar with control engineering. In this context the reader will benefit from a prior exposure to linear control systems from the "classical" point of view. This is the goal of the Section 2 which is an introduction of a lot of fundamental topics in control engineering such as feedback, Laplace transform, transfer function, Bode and Nyquist plots (Franklin et al. 1991; Dorf \& Bishop 1998) which are illustrated with case studies. In this section we also expose some elements for digital controlled systems such as sampled-data systems, $z$-transform and discrete time transfer function (Franklin et al. 1990; Astrom \& Wittenmark 2011) and we present basic case studies. Some paragraphs of the tutorial are selected passages or strongly inspired from the cited books. For instance the automobile cruise control example is presented in the book of Franklin et al. (1991). Our goal is not to teach the reader how to design linear controllers (several existing books do a good job for that) but rather to give a comprehensive understanding of feedback systems.

The third section is dedicated to the exposure of the Adaptive Optics control problem. The AO system is modeled as a multiple-input multiple-output (MIMO) feedback system using the "classical" control framework. A first category of control strategies: the optimized modal gain integral control (OMGI) proposed by Gendron \& Léna (1994) and its improvements is discussed, see (Dessenne et al. 1998). A static decoupling matrix is inserted in the feedback loop in order to divide the MIMO control problem in a series of single-input single-output (SISO) control problem. The design parameters are chosen to ensure stability and a trade-off between disturbance rejection and measurement noise amplification. The main quality of the optimized modal gain integral control, which is the current adaptive optics control system is to express some of the controller's signals in the modal base which facilitates the physical interpretation. Furthermore it is intrinsically a frequency approach: the analysis of the AO feedback system's performance is straightforward. The method can be used when the knowledge of the disturbance temporal dynamics is weak.

The last section contains the design of a controller from a modern control point of view (Kulcsár et al. 2006; Looze 2006). This approach was introduced for the first time by Paschall et al. (1991), which explicitly tries to minimize the mean-square residual wavefront error (and consequently maximize the imaging performance index as the Strehl ratio). This problem can be formulated as a linear quadratic Gaussian (LGQ) control problem, and the solution consists in the optimal state-feedback control of the DM and the optimal estimation of the atmospheric wavefront. The proposed approach emphasizes the ability of the LQG controller loop to reject the atmospheric aberration. We propose a generic second order autoregressive model to capture the main features of the aberrated wavefront. We derive a diagonal state space system which clearly separates the dynamics of the plant (DM \& WFS) from the disturbance dynamics (atmospheric model). Thus, we explicitly consider a disturbance rejection control problem, see (Bitmead et al. 1990), which facilitates the numerical resolution of the estimation problem: the order of the estimation discrete time algebraic Riccati equation is reduced. This point is important from a practical point of view for the new generation of 
AO systems exhibiting a large number of modes where control laws have to be designed in accordance with real time constraints. Numerical experiments using the Software Package CAOS have been conducted to demonstrate the effectiveness of the proposed approach.

\section{Classic feedback control: A tutorial}

\subsection{Definitions \& feedback framework}

\subsubsection{What is automatic control?}

Control is a general concept which refers to a specific interaction between two (or more) devices. Driving an automobile is a typical example: the driver has to control the vehicle to reach a given destination. In such a case, the car is manually controlled. At the opposite, automatic control only involves devices: this is the case of automobile cruise control. The rate flow of the fuel/air mixture is adjusted in real time depending on a speedometer measure to obtain a given speed.

\subsubsection{What is feedback?}

The main idea in control is feedback control where the variable/signal being controlled (speed, temperature...) is measured by a sensor and fed back to the process in order to influence the controlled signal. This feedback idea can be illustrated for the automobile cruise control and is described by a component block diagram in Figure 1. Main devices of the system are represented by blocks and arrows show interaction from one device to another.

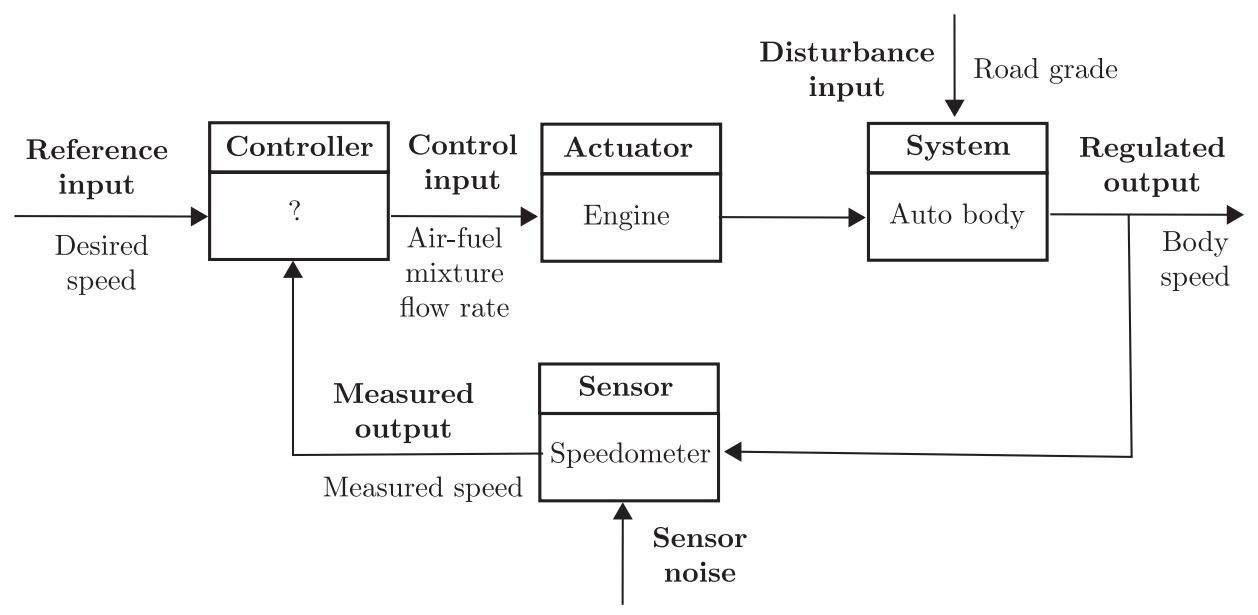

Fig. 1. Component block diagram of automobile cruise control.

Qualitatively, the temporal behavior of this controlled system can be analyzed. Suppose that when an air-fuel mixture is injected in the engine, the actual 
measured speed is below the desired speed. Then, the cruise controller will increase the air-fuel mixture flow rate causing an increase of the engine speed and consequently the body vehicle speed. If the actual speed is higher than the desired speed then the cruise controller will decrease the air-fuel mixture flow rate in order to reduce the body vehicle speed. For this example, the generic components of a classic feedback loop are shown in Figure 1. The main component is called the system (or plant or process) where one variable/signal is to be controlled or regulated. In our example the plant is the automobile body and the controlled/regulated output is the vehicle speed. The disturbance input is the road grade which acts on the system. The actuator is the component that influences the regulated variable: here the actuator is the engine. To obtain a feedback, we need to deliver to the controller a measured output which is provided by the sensor. In this case, the sensor is the speedometer. The role of the controller is to generate, using the reference input and the measured output, the control input. Feedback control properties can be demonstrated using quantitative analysis of a simplified model of the automobile cruise control. We will neglect the dynamic response of the car by considering only the steady state case. We will assume that for the range of speed of the vehicle, the approximated relations are linear. For the automobile speed, we measure speed on a level road at 55 kilometers per hour $(\mathrm{km} / \mathrm{h})$ and find that a unit change in our control (injection pump input) causes a $10 \mathrm{~km} / \mathrm{h}$ change in speed. When the grade changes by $1 \%$, we measure a speed change of $5 \mathrm{~km} / \mathrm{h}$. The accuracy of the speedometer is sufficient and can be considered exact. These relations permit to obtain the bloc diagram shown in Figure 2.

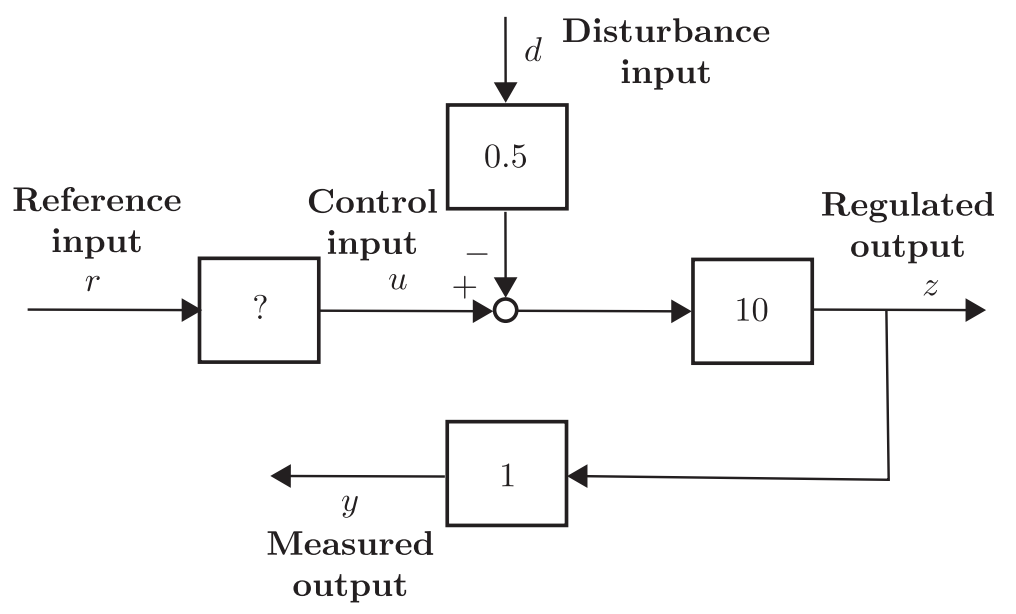

Fig. 2. Block diagram of automobile cruise feedforward control.

Here lines represent signals as regulated output $z$, control input $u$, disturbance input $d$, measured output $y$ and reference input $r$. Squared/round blocks represent respectively multiplication and summation. In Figure 2, the feedforward controller does not use the body speed. A possible control policy consists in inverting the 
plant characteristic and the controller sets $u=r / 10$. In this case we obtain the regulated output speed

$$
\begin{aligned}
z & =10(u-0.5 d) \\
& =10([r / 10]-0.5 d) \\
& =r-5 d .
\end{aligned}
$$

If $d=0$ (a level road) and $r=55$ then the vehicle speed will be $z=55$ and there will be no error. However if $d=1$ (a $1 \%$ grade) then the speed will be $r=50$ and we have a $5 \mathrm{~km} / \mathrm{h}$ error in speed.

In contrast to feedforward control, a feedback controller uses the measure of the controlled output (called the feedback signal) as in Figure 3 where the control input is $u=r-y=r-[0.9 z]$.

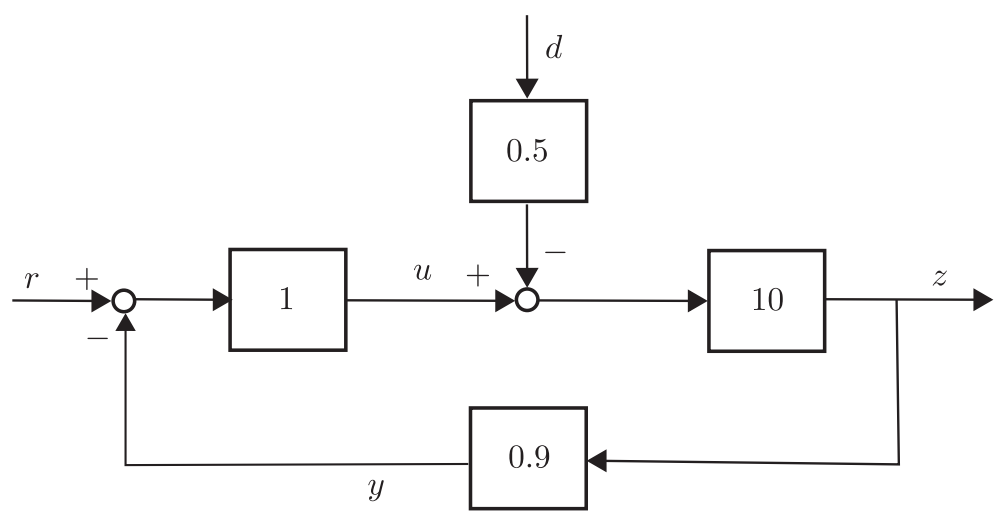

Fig. 3. Component block diagram of automobile cruise feedback control.

The topology of this block diagram include includes a loop: this is a closed loop control system. At the opposite the configuration shown in Figure 2 is called open loop control system. The equations of the closed loop control system are

$$
\begin{aligned}
z & =10(u-0.5 d) \\
& =10([r-0.9 z]-0.5 d) \\
& =10 r-9 z-5 w
\end{aligned}
$$

and finally

$$
z=r-d / 2 \text {. }
$$

In this case, if the reference speed is still $r=55$ and the grade $d=1$ then the vehicle speed will be $y=54.5$ and the error is $0.5 \mathrm{~km} / \mathrm{h}$. The effect of feedback is to reduce the speed error by a factor 10 ! If we include a gain factor for the controller greater than 1 the error will still decrease. But there is a limit for the gain value 
due to the power of the engine but more importantly because when the dynamics are introduced, feedback may induce poor temporal response (stability problems). As Stephen P. Boyd contends in (Boyd 1993), "a bad feedback controller can yield performance much worse than an open loop controller".

\subsubsection{Quantitative analysis in the time domain: A tentative}

In order to analyze a feedback controlled system we need to obtain a quantitative mathematical model of the plant. In this paper we assume that the process under study can be considered as linear over a reasonably large range of the signals and time invariant. That is, a mathematical model is frequently a set of ordinary differential equations and a specific solution can be found using a computer program. The output $s$ of a general time invariant linear system, in the time domain, is given by the convolution integral

$$
s(t)=(h * e)(t)=\int_{0}^{t} h(\tau) e(t-\tau) d \tau,
$$

where $e(t)$ is the input signal and where $h(t)$ is the impulse response. We can use the bloc diagram notation given in Figure 4.

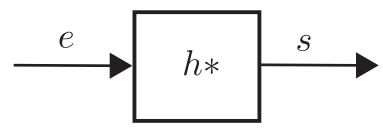

Fig. 4. Block diagram notation of the convolution operation.

This generic block diagram may describe every component of a feedback system as the controller, the actuator and the sensor. We note respectively $k, g_{1}$ and $g_{2}$ their impulse response. We study now a feedback system shown in Figure 5. The block diagram resembles an automobile cruise block diagram depicted in Figure 3. We require that the regulated output $z$ becomes zero: this is a disturbance rejection control problem. Thus the reference signal $r$ is zero and is not represented in the block diagram. We consider a more realistic model of the sensor: an additive sensor noise $n$ is taken into account. We will see later in the paper that this block diagram is a simplified model of an AO control loop.

The equation of the feedback system is

$$
z=d-\{\overbrace{g_{1} *[\underbrace{k *(\overbrace{g_{2} * z+n})}_{u}]}^{c}\},
$$

which can be rewritten as

$$
z=d-\left(g_{1} * k\right) * n-\left(g_{1} * k * g_{2}\right) * z .
$$




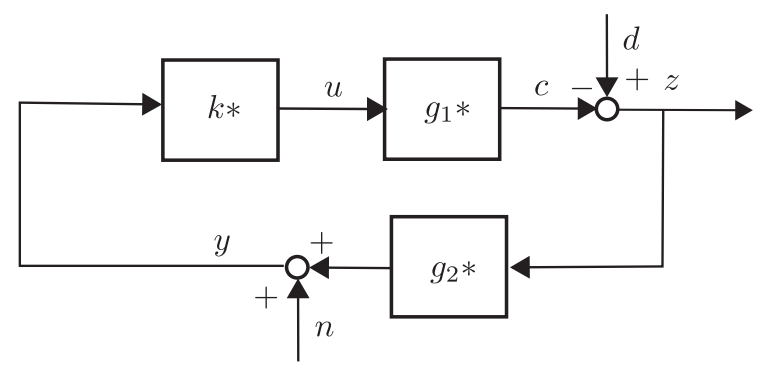

Fig. 5. Convolution based block diagram of the feedback system.

We have a complicated convolution Equation (2.3): the regulated output $z$ is the sum of the disturbance signal $d$, of the signal $\left(g_{1} * k\right) * n$ and of the signal $\left(g_{1} * k * g_{2}\right) * z$. This last signal is the response of the cascaded system with impulse response $g_{1} * k * g_{2}$ where the input is the regulated input $z$. The regulated output $z$ depends on itself: this is a feature of the feedback systems. In the time domain we have a complex convolution Equation (2.3) which is not easy to understand or to solve. We will see that in the frequency domain the computation and the interpretation of the transformed equation is straightforward.

\subsection{Feedback systems: A frequency approach}

\subsubsection{Laplace transform \& transfer functions}

The Laplace transform is well suited to find the solution of Equation (2.2) and to give interesting information (settling time, overshoot, final value) of feedback systems. The Laplace transform of a signal $f(t)$ is defined as

$$
\mathcal{L}\{f\}(s)=\int_{0}^{\infty} f(t) e^{-s t} d t
$$

A straightforward consequence of convolution integral (2.1) is

$$
\mathcal{L}\{s\}(s)=H(s) \mathcal{L}\{e\}(s),
$$

where $H(s)=\mathcal{L}\{h\}(s)$ is called the transfer function. Thus the Laplace transform of the output $\mathcal{L}\{s\}$ is the product of the transfer function $H$ and of the Laplace transform $\mathcal{L}\{e\}$. In the frequency domain Equation (2.5) is the counterpart of convolution integral (2.1) in the time domain. We can use the bloc diagram notation given in Figure 4.

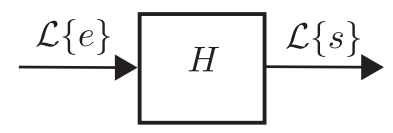

Fig. 6. Block diagram notation of the transfer function. 


\subsubsection{Feedback system's transfer functions}

The feedback system shown in Figure 5 can be "translated" in the frequency domain. We call $G_{1}(s)=\mathcal{L}\left\{g_{1}\right\}(s), G_{2}(s)=\mathcal{L}\left\{g_{2}\right\}(s)$ and $K(s)=\mathcal{L}\{k\}(s)$ respectively the actuator transfer function, the sensor transfer function and the controller transfer function. The block diagram is drawn again: the controller's block is moved at the bottom and the sensor's block is displaced at the top.

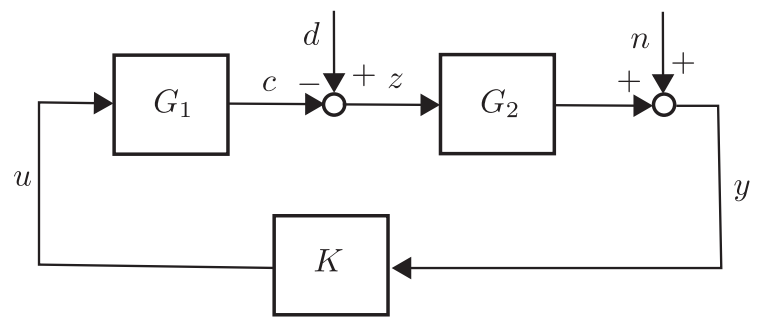

Fig. 7. Transfer function based block diagram of the feedback system.

In the frequency domain blocks $G_{1}(s), G_{2}(s)$ and $K(s)$ are simple scaling systems. From block diagram in Figure 7 we obtain

$$
\mathcal{L}\{z\}=\mathcal{L}\{d\}-\{\overbrace{G_{1}[\underbrace{K(\overbrace{G_{2} \mathcal{L}\{z\}+n}^{\mathcal{L}\{y\}}]}_{\mathcal{L}\{u\}}]}^{\mathcal{L}\{c\}},
$$

which can be solved as

$$
\mathcal{L}\{z\}=\underbrace{\frac{1}{1+G_{1} K G_{2}}}_{S} \mathcal{L}\{d\}-\underbrace{\frac{G_{1} K}{1+G_{1} K G_{2}}}_{T} \mathcal{L}\{n\} .
$$

To understand how controllers ensure relevant properties for the feedback system, the Equation (2.6) is central. We call

$$
L=G_{1} K G_{2}
$$

the loop transfer function,

$$
S=\frac{1}{1+L}
$$

the sensitivity transfer function, and

$$
T=G_{1} K S
$$

the noise sensitivity transfer function. For "ideal control" we want $z=0$ and consequently

$$
\mathcal{L}\{z\} \approx 0 \mathcal{L}\{d\}+0 \mathcal{L}\{n\} .
$$


Disturbance rejection is achieved when $S \approx 0$ and noise rejection is ensured when $T \approx 0$. In practice these two transfer function $S$ and $T$ cannot be small at the same values of $s$ and a trade off should be achieved during the design of the controller transfer function $K$.

\subsection{Standard examples}

In this section we present two case studies to illustrate the concepts introduced in the preceding paragraph. We will also study the properties ensured both in the frequency domain and in the time domain for classical controllers (proportional and integral).

\subsubsection{Case study 1}

We suppose that the actuator and the sensor have instantaneous responses:

$$
G_{1}(p)=\alpha, G_{2}(p)=\beta,
$$

where $\alpha$ and $\beta$ are fixed positive scalar. We use a proportional controller which produces the control input

$$
u(t)=k_{P} y(t)
$$

where the scalar $k_{P}$ is the proportional gain. We also consider an integral controller which imposes the control input

$$
u(t)=k_{I} \int_{0}^{t} y(\tau) d \tau
$$

where the parameter $k_{I}$ is the integrator gain. Time domain Equations (2.12) and (2.13) can be cast under the convolution integral form $k * y$ with impulse response $k(t)=k_{P} \delta(t)$ and $k(t)=k_{I}$. Hence, controller transfer function $K$ can be calculated. For numerical purpose, we set the actuator's gain $\alpha=10$ and the sensor's gain $\beta=1$. We consider a proportional controller with the gain $k_{P}=0.2$, an integral controller with the gain $k_{I}=0.4$ and another integral controller with the gain $k_{I}=1$. These controllers are

$$
K^{(a)}(s)=0.2, \quad K^{(b)}(s)=\frac{0.4}{s}, \quad K^{(c)}(s)=\frac{1}{s} .
$$

The corresponding sensitivity transfer function, which we denote $S^{(a)}(s), S^{(b)}(s)$, and $S^{(c)}(s)$ respectively, can be computed from (2.8). The closed-loop systems that result from using the controllers $K^{(a)}, K^{(b)}$, and $K^{(c)}$ can be compared by examining the sensitivity transfer function $S^{(a)}, S^{(b)}$, and $S^{(c)}$. The magnitudes $\left|S^{(a)}(j \omega)\right|,\left|S^{(b)}(j \omega)\right|$, and $\left|S^{(c)}(j \omega)\right|$ are plot in Figure 8a. From this figure we can conclude that a low frequency disturbance input will have the least effect in the feedback system with controller $K^{(c)}$ i.e. the best disturbance rejection performance. The real disturbance input is usually unknown. A reasonable approach is 
to choose a standard test input signal as a step $d(t)=1$ shown in Figure 9a. This step response checks the ability of the system to perform under normal operating conditions using generic test input signals as a step $d(t)=1$ shown in Figure 9a. The step responses of the sensitivity transfer function are shown in Figure 8b. From this figure it can be seen that the controller $K^{(c)}$ ensures the faster decay of the transient response.

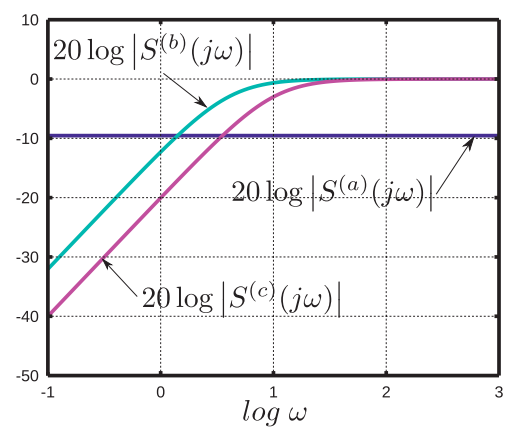

(a)

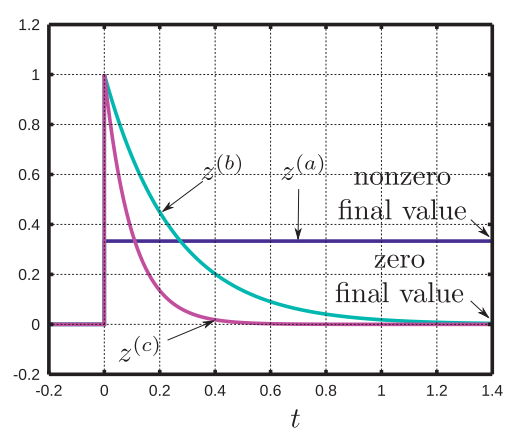

(b)

Fig. 8. (a) Magnitude of the sensitivity transfer functions $S^{(a)}, S^{(b)}$, and $S^{(c)}$. (b) The step responses from disturbance input $d$ to regulated output $z$ for the sensitivity transfer functions $S^{(a)}, S^{(b)}$, and $S^{(c)}$.

The step responses from the disturbance input $d$ to the control input $z$ for the three feedback systems are shown in Figure 9b. For integral controllers $K^{(b)}$ and $K^{(c)}$, final value of their output (control input) is zero when final value of the regulated output $z$ is zero. This is an important feature of integral controllers which ensures zero steady-state error for the actuator/plant/sensor configuration given in (2.11).

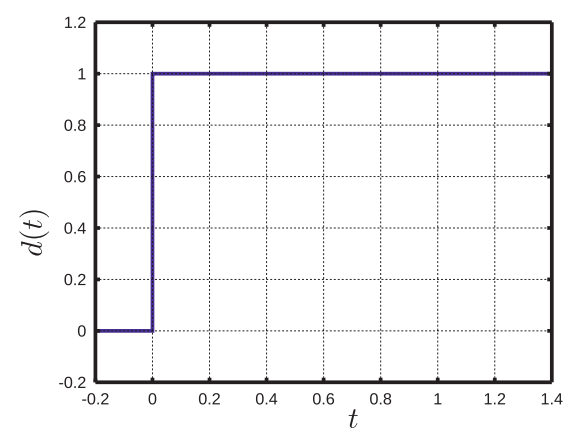

(a)

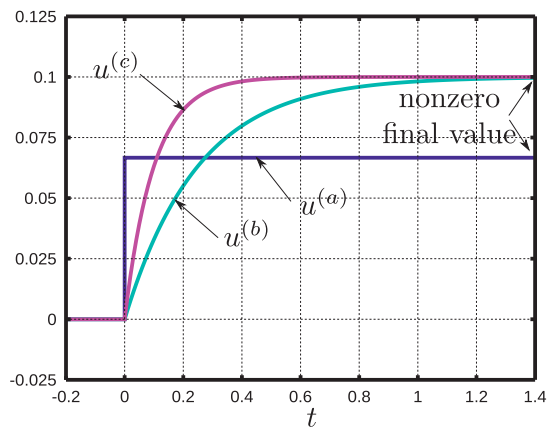

(b)

Fig. 9. (a) A step signal $d$. (b) The step responses from disturbance input $d$ to control input $z$ for the transfer functions $K^{(a)} S^{(a)}, K^{(b)} S^{(b)}$, and $K^{(c)} S^{(c)}$. 


\subsubsection{Case study 2}

In this section we still consider the standard closed loop system shown in Figure 7. The sensor always has an instantaneous response but here the actuator is a second order dynamical system

$$
G_{1}(p)=\alpha \frac{\omega_{n}^{2}}{p^{2}+2 \zeta \omega_{n} p+\omega_{n}^{2}}, \quad G_{2}(p)=\beta .
$$

For a numerical purpose, we conserve the actuator's gain $\alpha=10$ and the sensor's gain $\beta=1$ of Section 2.3.1. We set the damping factor $\zeta=0.7$ and the natural frequency $w_{n}=10$. The controller transfer functions are given in (2.14). The corresponding loop transfer function, which we denote $L^{(d)}(s), L^{(e)}(s)$, and $L^{(f)}(s)$ respectively, can be computed from (2.7). The same notation holds for

- the sensitivity transfer function $S^{(d)}(s), S^{(e)}(s), S^{(f)}(s)$ calculated from (2.8);

- the noise sensitivity transfer function $T^{(d)}(s), T^{(e)}(s), T^{(f)}(s)$ computed from (2.9).

The magnitudes $\left|S^{(d)}(j \omega)\right|,\left|S^{(e)}(j \omega)\right|$, and $\left|S^{(f)}(j \omega)\right|$ are plot in Figure 10a. These plots should be compared to the plots depicted in Figure 8a. From this figure we can conclude that a low frequency disturbance input will have the least effect on the feedback system with controller $K^{(c)}$ i.e. the best disturbance rejection performance. In the low frequencies domain the remarks in Section 2.3.1 should be similar but there is a large peak of the magnitude $\left|S^{(f)}(j \omega)\right|$. We can conclude that the feedback system with controller $K^{(c)}$ is not stable enough.

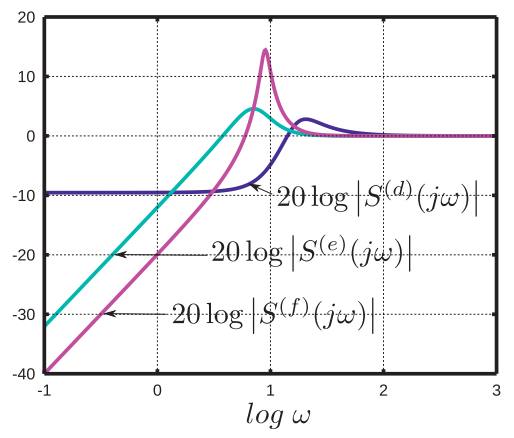

(a)

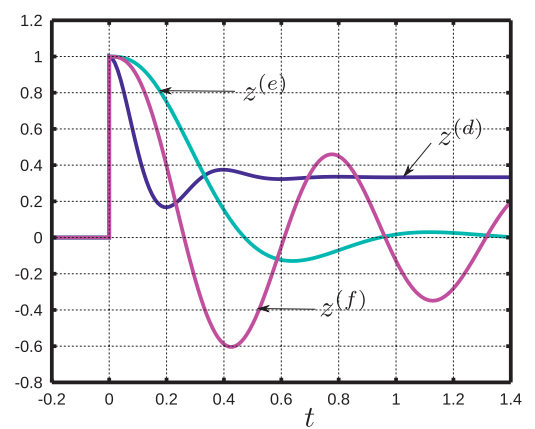

(b)

Fig. 10. (a) Magnitude of the sensitivity transfer functions $S^{(a)}, S^{(b)}$, and $S^{(c)}$. (b) The step responses from disturbance input $d$ to regulated output $z$ for the sensitivity transfer functions $S^{(a)}, S^{(b)}$, and $S^{(c)}$.

The Nyquist plots of the loop transfer function $L^{(d)}, L^{(e)}$ and $L^{(f)}$ are shown in Figure 11. The Nyquist plot of $L^{(f)}(j \omega)$ is too close to the -1 point, see 
(Franklin et al. 1991). We can corroborate that the stability margins are small for the feedback system with the controller $K^{(c)}$. The step responses of the sensitivity transfer function are shown in Figure 10b. From this figure it can be seen that the controller $K^{(c)}$ has a poor transient response. $z$ plot exhibits oscillatory behavior: the damping ratio of the feedback system is weak. Thus controller $K^{(b)}$ is selected to be the operating controller.

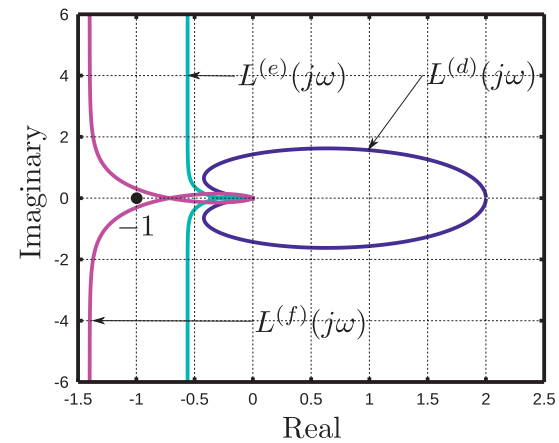

(a)

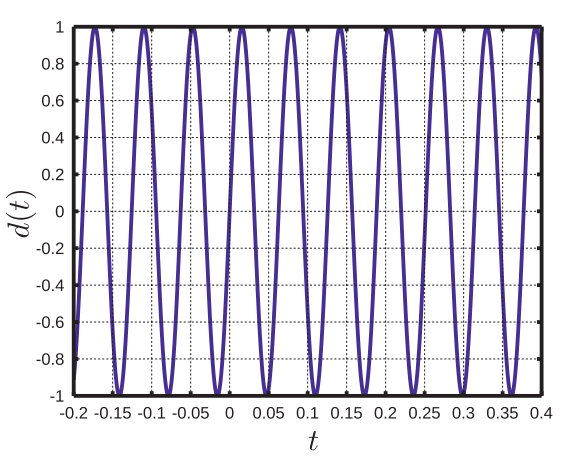

(b)

Fig. 11. (a) Nyquist plot for the loop transfer functions $L^{(d)}(s), L^{(e)}(s)$ and $L^{(f)}(s)$. (b) A sinusoidal signal $d$.

To assess the noise rejection performance we plot the magnitude of the noise sensitivity transfer function $T^{(d)}, T^{(e)}$ and $T^{(f)}$. Figure 12 shows $\left|T^{(d)}(j \omega)\right|$, $\left|T^{(e)}(j \omega)\right|$, and $\left|T^{(f)}(j \omega)\right|$, i.e., the magnitudes of the feedback system transfer functions from measurement noise $n$ to regulated output $z$. From this figure, we can conclude that a high frequency sensor noise will have the greatest effect on $z$ with the controller $K^{(a)}(s)$ and the least effect with the controller $K^{(b)}(s)$. For a given controller, for instance $K^{(b)}(s)$, remark that the magnitude $\left|S^{(e)}(j \omega)\right|$ and $\left|T^{(e)}(j \omega)\right|$ cannot be small in the same frequency domain.

The response of the noise sensitivity transfer function from a sinusoidal disturbance input $d$ plotted in Figure $11 \mathrm{~b}$ are shown in Figure 10b. From this figure it can be seen that the sinusoidal steady-state response of the feedback system with controller $K^{(b)}(s)$ is the smallest. Controller $K^{(b)}(s)$ ensures the best noise rejection performance.

\subsection{Digital controlled systems}

\subsubsection{Sampled-data feedback system}

In practice all control systems that are implemented today are based on a digital computer. A computer controlled system is sketched schematically in Figure 13. This block diagram is very similar to block diagram depicted in Figure 7, except for a digital device which generates the control action. The analog-to-digital $(A / D)$ 


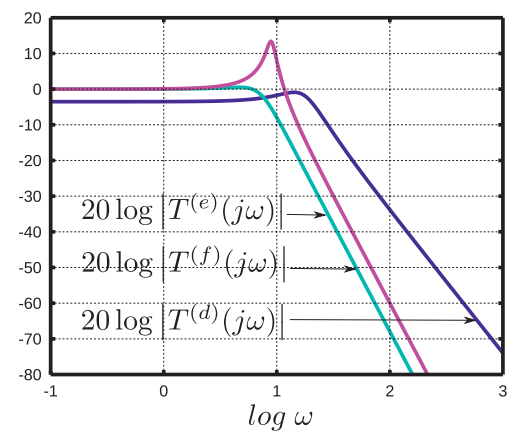

(a)

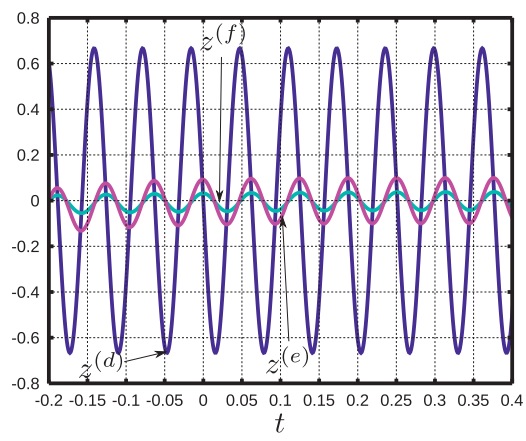

(b)

Fig. 12. (a) Magnitude of the noise sensitivity transfer functions $T^{(a)}, T^{(b)}$, and $T^{(c)}$. (b) The responses from sinusoidal disturbance input $d$ to regulated output $z$ for the sensitivity transfer functions $T^{(a)}, T^{(b)}$, and $T^{(c)}$.

converter shown in Figure 13 is a device that converts the sensor output $y(t)$ to digital numbers read by the computer. We assume that all the numbers arrive with the same fixed period $T$ and we neglect the quantization operation thus

$$
y(k)=\left.y(t)\right|_{t=k T} .
$$

The computer interprets the converted signal, $y(k)$ as a sequence of numbers, processes the measurements using an algorithm, and provides a new sequence of numbers $u(k)$. The digital-to-analog $(D / A)$ converter converts the sequence of number $u(k)$ to the physical control signal $u(t)$. In many case the signal $u(t)$ is kept constant between the successive sampling instants

$$
u(t)=u(k) \quad k T \leq t<(k+1) T .
$$

We call variables $y(k)$ and $u(k)$ discrete time signals to distinguish them from continuous time signals $y(t)$ and $u(t)$ which change continuously in time. The computer-controlled system contains both continuous-time signals and discretetime signals and is called a sampled-data system.

For a numerical purpose, we assume that the actuator and the sensor are fading memory systems with transfer function

$$
G_{1}(p)=\frac{\alpha}{0.1 s+1}, \quad G_{2}(p)=\frac{\beta}{0.1 s+1},
$$

and we retain the actuator's gain $\alpha=10$ and the sensor's gain $\beta=1$ of Section 2.3.1. The sampling period is $T=0.2$ and the disturbance input is a step $d(t)=1$ shown in Figure 9a. The control sequence $u(k)$ is obtained from the measurement sequence $y(k)$ using the control algorithm

$$
u(k)=u(k-1)+k_{I} T y(k),
$$




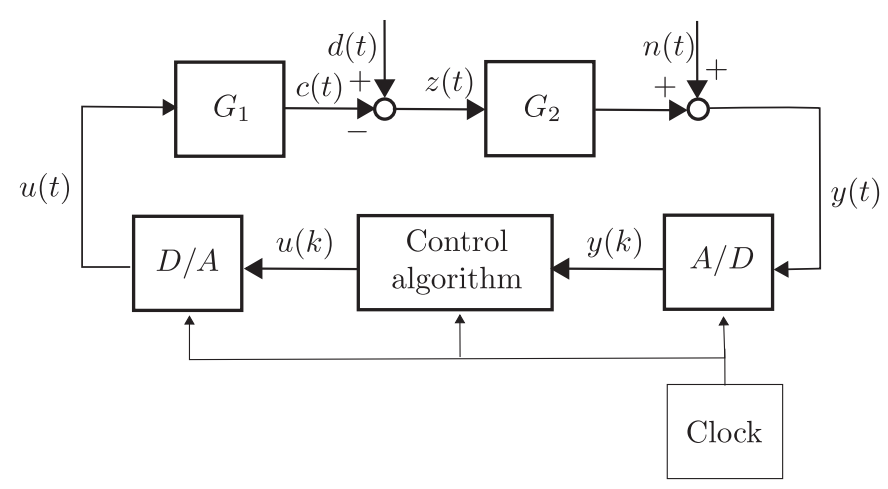

Fig. 13. Sampled-data feedback system.

where $k_{I}=0.5$. The behavior of the A/D converter is illustrated in Figure 14 . Figure 15a is a plot of the sequence of numbers $u(k)$ obtained from the sequence of numbers $y(k)$ plotted in Figure 14a. Note that the D/A converter keeps the signal $u(t)$ constant between the successive sampling instant $k T$, see the Figure 15b.

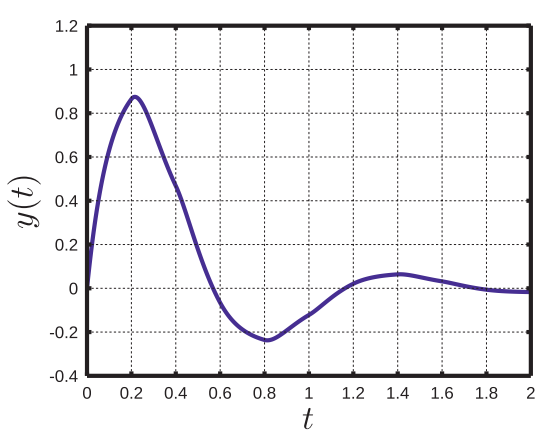

(a)

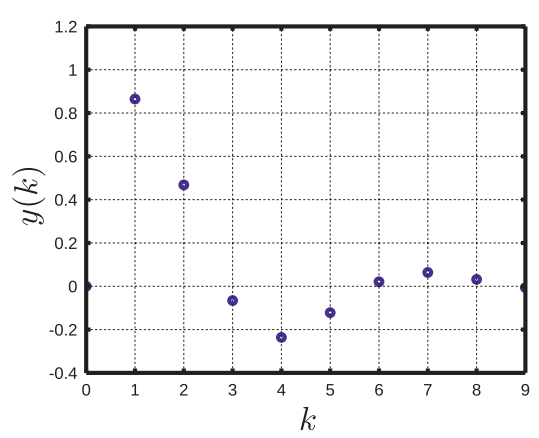

(b)

Fig. 14. Analog-to-digital (A/D) converter operation: (a) measured output $y(t)$, (b) control algorithm input $y(k)$.

For the sake of brevity we do not discuss sampling and reconstruction of continuous-time signals. For a comprehensive exposure, the interested reader may consult the book of Astrom \& Wittenmark (2011). Remark that to avoid aliasing effect, it is necessary to filter the analog signal $y(t)$ before the A/D converter so that the signal obtained do not have frequencies above the Nyquist frequency. Note that the output of the D/A are rectangular pulses which causes multiple harmonics above the Nyquist frequency. This may cause difficulties for systems with weakly damped oscillatory modes. If needed, the multiple harmonics could be removed with a low pass filter acting as a reconstruction filter. The overall 


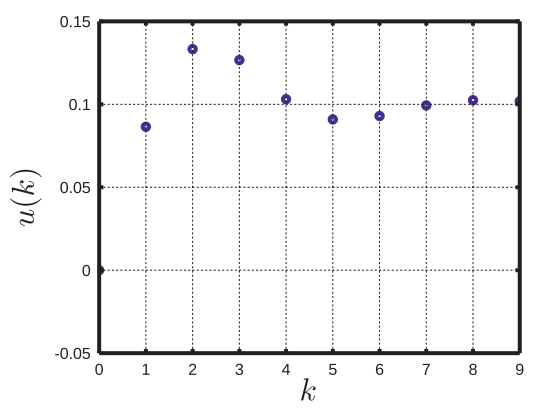

(a)

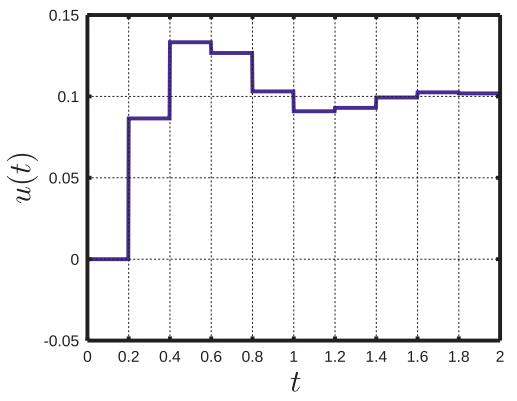

(b)

Fig. 15. Digital-to-analog (D/A) converter operation: (a) control algorithm output $u(k)$, (b) control input $u(t)$.

behavior of this hybrid feedback system which incorporates both continuous time signals and discrete time signals can be studied by two different approaches.

1. The first approach, called the emulation design method, see (Franklin et al. 1991), deals with continuous time transfer function. In this case the digital computer behavior shown in Figure 16 is approximated by an equivalent continuous time system described by transfer function $K(s)$, see Figure 17 . The overall feedback system is assumed to be continuous and the continous time framework presented in Sections 2.3.1 and 2.3.2 can be used considering the feedback loop depicted in Figure 7. This approach is discussed in Section 2.4.2.

2. For the latter approach the sampled-data feedback system is transformed into a discrete time feedback system. For this purpose the continuous part of the system is sampled as seen from the digital computer's point of view. The resulting feedback system is characterized by a discrete time transfer function using the $z$-transform. In this case discrete time controller design methods may be used. An analysis of the feedback discrete time system is performed in Section 2.4.3.

\subsubsection{Emulation design method}

The output of an integral controller (2.13) at time $t=k T$ is

$$
\begin{aligned}
u(k T) & =k_{I} \int_{0}^{k T} y(\tau) d \tau \\
& =k_{I} \int_{0}^{k T-T} y(\tau) d \tau+k_{I} \int_{k T-T}^{k T} y(\tau) d \tau \\
& =u(k T-T)+k_{I} \underbrace{\int_{k T-T}^{k T} y(\tau) d \tau}_{I} .
\end{aligned}
$$


Several approximations of the incremental term $I$ can be chosen as for instance the backward rectangular rule $I \approx T y(k T)$. Hence we obtain

$$
\underbrace{u(k T)}_{u(k)}=\underbrace{u(k T-T)}_{u(k-1)}+k_{I} T \underbrace{y(k T)}_{y(k)},
$$

which is equivalent to Equation (2.19). Thus the digital computer with algorithm defined by Equation (2.19) is a discrete time equivalent to the continuous time controller $K(s)=k_{I} / s$.

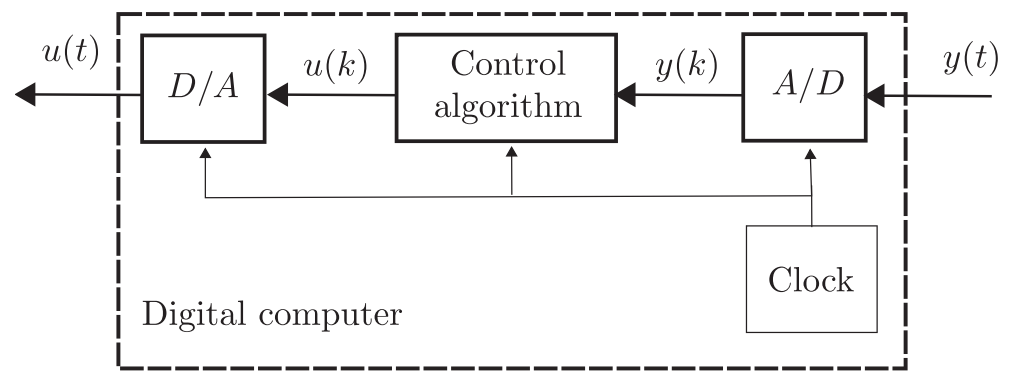

Fig. 16. Association of the $A / D$ converter with the control algorithm and with the D/A converter.

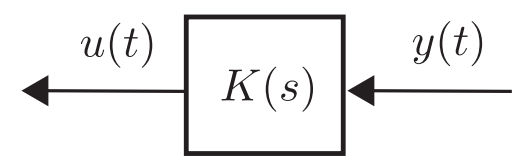

Fig. 17. Equivalent transfer function $K(s)$.

We consider that the feedback system is described by the block diagram shown in Figure 7. For the given transfer functions $G_{1}, G_{2}$ defined by (2.18), standard continuous time design method can be used to obtain the integral controller

$$
K^{(h)}(s)=\frac{0.5}{s}
$$

This continuous time controller is approximated with the difference Equation (2.19) and we call $K^{(i)}$ and $K^{(j)}$ the discrete time controller with the sampling period $T=0.2$ and $T=0.05$. We assume that the disturbance input $d$ is a step. We called $z^{(h)}$ the "ideal" regulated output response of the continuous time feedback system, $z^{(i)}$ the regulated output response of the sampled-data feedback system when the discrete time controller is $K^{(i)}$, and $z^{(j)}$ the regulated output response of the sampled-data feedback system when the discrete time controller is $K^{(j)}$. These signals are plotted in Figure 18a an Figure 19a. From these figures we can conclude that $z^{(j)}$ is the best approximation of the "ideal" regulated output response $z^{(h)}$. 


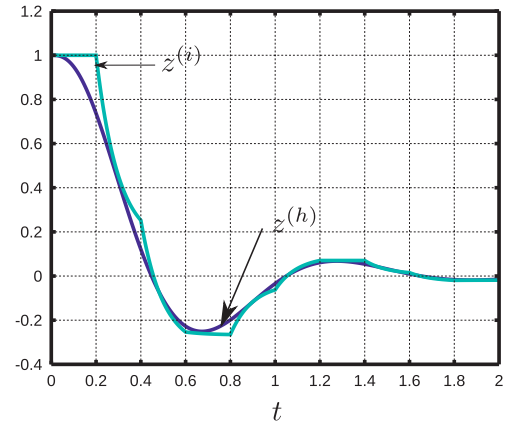

(a)

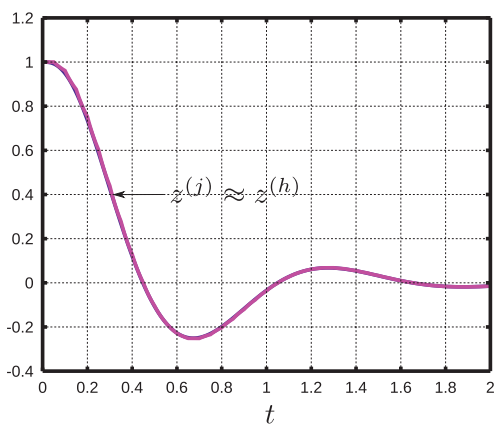

(b)

Fig. 18. "Ideal" regulated output response $z^{(h)}$ of the continuous time feedback system, regulated output response $z^{(i)}$ of the sampled-data feedback system when $T=0.2$, and regulated output response $z^{(j)}$ of the sampled-data feedback system when $T=0.05$.

The "ideal" input response $u^{(h)}$ of the continuous time feedback system, the regulated output response $u^{(i)}$ of the sampled-data feedback system when the discrete time controller is $K^{(i)}$, and the regulated output response $u^{(j)}$ of the sampled-data feedback system when the discrete time controller is $K^{(j)}$ are shown in Figure $18 \mathrm{~b}$ and in Figure 19b. It can be seen that the response $u^{(j)}$ matches the "ideal" response $u^{(h)}$. We can conclude that clearly the sampling period $T=0.2$ is too rough and that the sampling period $T=0.05$ ensures a satisfactory performance. As mentioned by Franklin et al. (1991), "sampling at a rate that is over 20 times faster than the bandwidth is a good, safe rule of thumb".

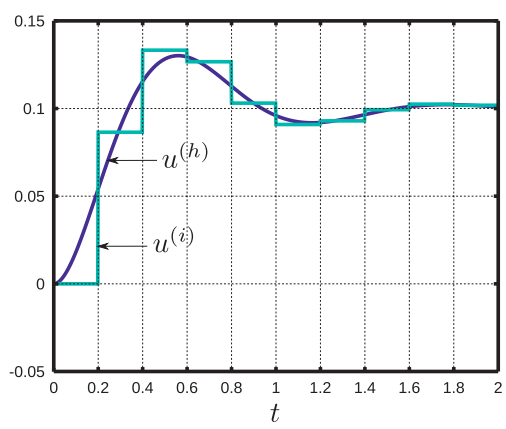

(a)

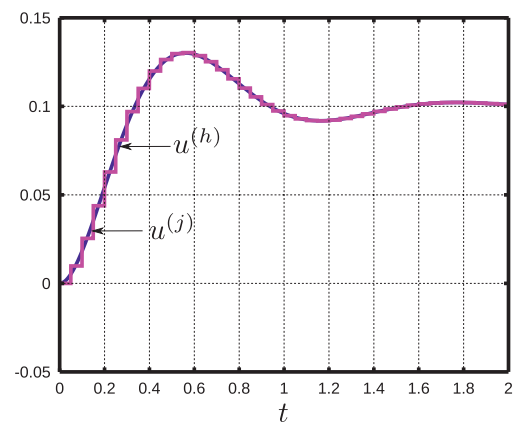

(b)

Fig. 19. "Ideal" regulated output response $u^{(h)}$ of the continuous time feedback system, regulated output response $u^{(i)}$ of the sampled-data feedback system when $T=0.2$, and regulated output response $u^{(j)}$ of the sampled-data feedback system when $T=0.05$. 


\subsubsection{Discrete time controller design}

In Figure 13 the job of the digital computer is to take the sampled value $y(k)$ and to compute the values $u(k)$ to be sent to the $\mathrm{D} / \mathrm{A}$ converter. The treatment of the data inside the computer can be expressed as a linear difference equation as for example the Equation (2.19), which describes a discrete time invariant linear system. In Section 2.2 the Laplace transform plays an important role and permits to introduce the transfer function and frequency interpretation of the closed loop system shown in Figure 7. The discrete-time analog of the Laplace transform is the $z$-transform which is a convenient tool to study general discrete linear systems. The $z$-transform of a signal $y(k)$ is defined as

$$
\mathcal{Z}\{y\}(z)=\sum_{k=0}^{\infty} y(k) z^{-k}
$$

where $z$ is a complex variable. If we multiply (2.19) by $z^{-k}$ and sum over $k$ we obtain

$$
\underbrace{\sum_{k=0}^{\infty} u(k) z^{-k}}_{\mathcal{Z}\{u\}(z)}=\sum_{k=0}^{\infty} u(k-1) z^{-k}+k_{I} T(\underbrace{\sum_{k=0}^{\infty} y(k) z^{-k}}_{\mathcal{Z}\{y\}(z)}) .
$$

In the first term on the right hand side, we let $k-1=j$ to get $\sum_{k=0}^{\infty} u(k-1) z^{-k}=$ $\sum_{j=1}^{\infty} u(j) z^{-(j+1)}=z^{-1} \mathcal{Z}\{u\}$. Equation (2.20) can be can be rewritten as

$$
\mathcal{Z}\{u\}(z)=z^{-1} \mathcal{Z}\{u\}(z)+k_{I} T \mathcal{Z}\{y\}(z)
$$

which is simply an algebraic equation in $z$. The solution is

$$
\mathcal{Z}\{u\}(z)=\underbrace{k_{I} T \frac{z}{z-1}}_{K(z)} \mathcal{Z}\{y\}(z) .
$$

We have obtained

$$
\mathcal{Z}\{u\}(z)=K(z) \mathcal{Z}\{y\}(z)
$$

where $K(z)=k_{I} T \frac{z}{z-1}$ is called the discrete time transfer function. Thus the $z$-transform of the output $\mathcal{Z}\{u\}$ is the product of the transfer function $K$ and the $z$-transform $\mathcal{Z}\{y\}$. We can use the bloc diagram notation given in Figure 20 .

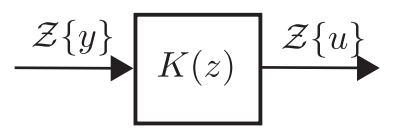

Fig. 20. Block diagram notation of the transfer function.

All the framework presented in Section 2.3 for analyzing continuous time systems can be extended to discrete time systems. We consider a discrete time system 
with the associated block diagram shown in Figure 21 where $K(z)$ is the controller transfer function, $G_{1}(z)$ is the actuator transfer function, and $G_{2}(z)$ is the sensor transfer function. This block diagram is similar to the block diagram depicted in Figure 7.

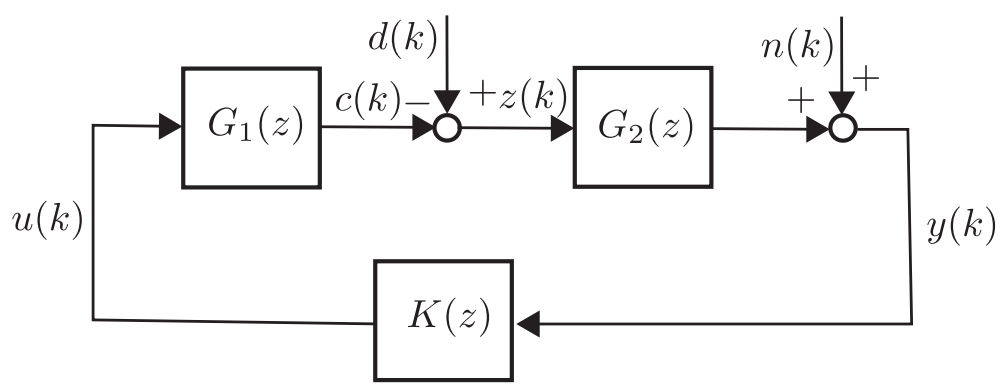

Fig. 21. Discrete time feedback system.

Hence the regulated output response is

$$
\mathcal{Z}\{z\}=\underbrace{\frac{1}{1+G_{1}(z) K(z) G_{2}(z)}}_{S(z)} \mathcal{Z}\{d\}-\underbrace{\frac{G_{1}(z) K(z)}{1+G_{1}(z) K(z) G_{2}(z)}}_{T(z)} \mathcal{Z}\{n\} .
$$

We still use the following terminology: (i) $L(z)=G_{1}(z) K(z) G_{2}(z)$ is the loop transfer function; (ii) $S(z)=\frac{1}{1+L(z)}$ is the sensitivity transfer function; (iii) $T(z)=G_{1}(z) K(z) S(z)$ is the noise sensitivity transfer function. All results presented in Section 2.3 for continuous time feedback systems are relevant for discrete time feedback systems.

The main difficulty concerns the correspondence between this block diagram shown in Figure 21 and the block diagram of the "real" sampled data feedback system depicted in Figure 13. This block diagram is redrawn in Figure 22 to make the comparison easier.

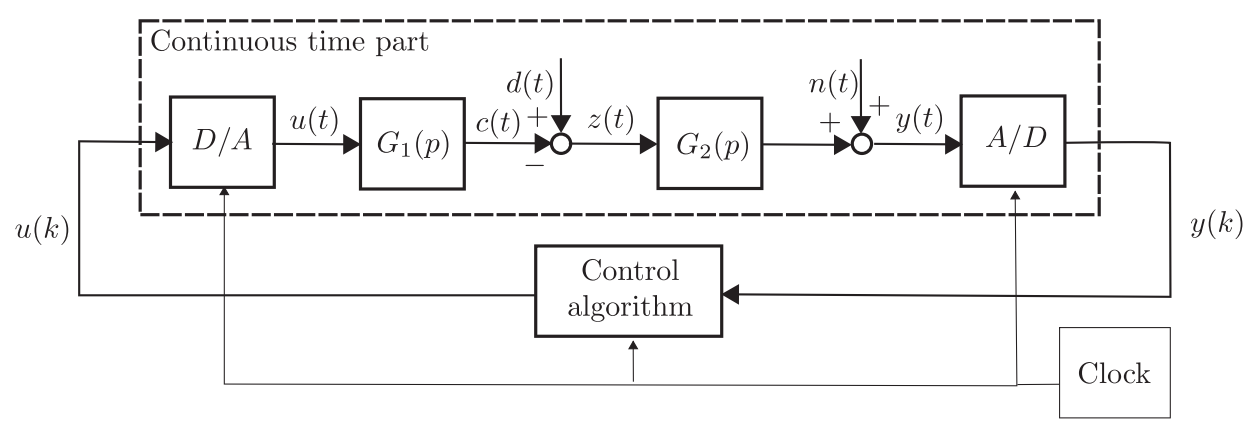

Fig. 22. Sampled-data feedback system. 
It is obvious to note that the control algorithm is represented by the transfer function $K(z)$. For a "perfect" connection: (i) the discrete time transfer function $G_{1}(z)$ should be viewed as the composition of the D/A converter system and the actuator transfer function $G_{1}(p)$; (ii) the discrete time transfer function $G_{2}(z)$ should describe the actuator transfer function $G_{2}(p)$ and the A/D converter. In general this connection is not possible and a deeper analysis should be performed using the pulse transfer function formalism, see (Franklin et al. 1990; Astrom \& Wittenmark 2011) which is beyond the scope of this tutorial. However in the absence of continuous time disturbance $d$, the discretization of the continuous time part of sampled data feedback system is a standard result, see Franklin et al. (1990) and allows to obtain the aggregated/global transfer function $G_{1}(z) G_{2}(z)$. But this global transfer function cannot be split in order to obtain transfer function $G_{1}(z)$ and transfer function $G_{2}(z)$. Yet for some special case of sensor transfer function $G_{2}(p)$ as CCD-based sensor, see (Looze 2005), the connection of the sampled data feedback system's block diagram shown in Figure 22 and the discrete time feedback system's block diagram shown in Figure 21 is faithful.

\section{Adaptive optics feedback control}

\subsection{Problem statement and wavefront spatial discretization}

Among its applications, AO systems can be used to reduce the effects of atmospheric turbulence on images taken from ground-based telescopes. The principle of a classical AO system is depicted in Figure 23. The atmospheric wavefront on the telescope aperture, defined at instant $t$ as the two dimensional function $\psi_{a}(x, t)$, is the input of the feedback system. The deformable mirror introduces a correction denoted by $\psi_{m}(x, t)$ which is subtracted from the incoming/atmospheric wavefront to obtain the outcoming/residual wavefront

$$
\psi_{r}(x, t)=\psi_{a}(x, t)-\psi_{m}(x, t)
$$

The shape of the DM is adjusted in real time using the measurements $y$ of a wavefront sensor which provides the local slopes of the residual wavefront, see Figure 24.

There exists different type of deformable mirrors and we choose to study the case of the most common one. For additional details on basic principles of adaptive optics, the reader can consult (Roddier 1999). We assume that the frequency bandwidth of the DM is higher than the bandwidth of the A0 loop. Moreover the DM's deformation is sufficiently small to consider a linear response. $n_{u}$ actuators are used and we denote $a_{i}(t)$ the stroke of the ith actuator. Thus the DM's shape is modeled as follows

$$
\psi_{m}(x, t)=\sum_{i=1}^{n_{u}} a_{i}(t) f_{i}(x),
$$




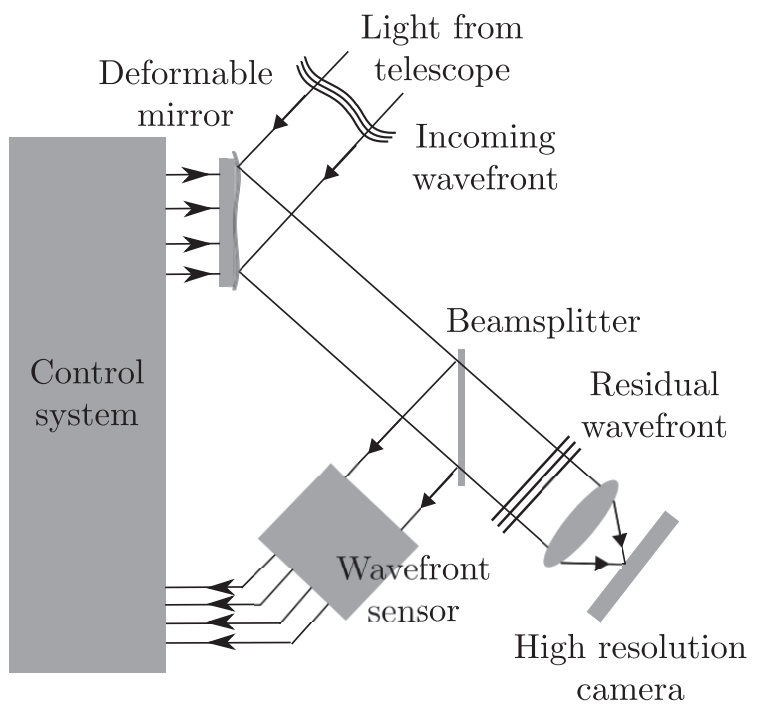

Fig. 23. Adaptive optics system.

where $f_{i}(x)$ is called the influence function of the ith actuator. We suppose that the DM's actuators and the associated power amplifiers have sufficient fast dynamics such that we assume that

$$
a_{i}(t)=u_{i}(t)
$$

We denote $u_{i}(t)$ the control input which is the power amplifier input of the ith actuator.

Different types of sensors (curvature sensor, pyramid wavefront sensor) may be used to estimate the distortions affecting the outcoming wavefront but the most frequently encountered in existing applications is the Shack-Hartmann (SH) wavefront sensor. The principle of a SH wavefront sensor is shown in Figure 24. The outcoming wavefront is imaged using a lenslet array of size $n_{w}$. Each lens takes a small part of the aperture, called sub-pupil, and forms an image of the source recorded by the detector, typically a CCD. If no wavefront aberrations are present, the image pattern is a grid of spots with constant intervals. As soon as the wavefront is distorted, the images are displaced from their nominal positions. Displacements of image centroids in two orthogonal directions $u, v$ are proportional to the average wavefront slopes in $u, v$ over the subapertures. The shift is computed using classic methods (center of gravity algorithms, ...). Thus, a $\mathrm{SH}$ sensor measures the wavefront average slopes $\left(\alpha_{u, i}, \alpha_{v, i}\right)$ for each subaperture $i$.

A usual representation of wavefront is made through the orthogonal basis, typically Karhunen-Loève functions or Zernike polynomials as defined in (Noll 1976). An infinite number of functions is required to characterize the wavefront, but a truncated basis $\left\{F_{i}(x)\right\}$ of dimension $n_{b}$, that we called the modal basis is used for 
incoming wavefront

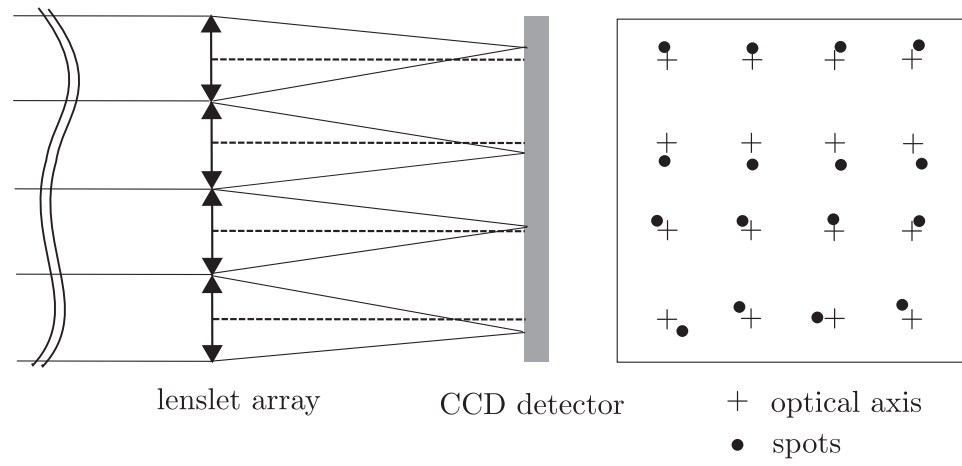

Fig. 24. Shack-Hartmann wavefront sensor principle.

implementation purpose. Thus the atmospheric wavefront $\psi_{a}$ can be decomposed on the modal basis as follows:

$$
\psi_{a}(x, t) \approx \sum_{i=1}^{n_{b}} w_{a, i}(t) F_{i}(x),
$$

where we denote $w_{a, i}$ the modal coordinates which are the coefficients of this decomposition. We collect the scalar coefficient signals $w_{a, 1}, \ldots, w_{a, n_{b}}$ to form the vector

$$
w_{a}(t)=\left[\begin{array}{c}
w_{a, 1}(t) \\
\vdots \\
w_{a, n_{b}}(t)
\end{array}\right] .
$$

The same representation (3.4) is used for the mirror correction $\psi_{m}$, and the residual wavefront $\psi_{r}$; similarly the coefficient signals are collected to form vector signals $w_{m}$ and $w_{r}$. Control inputs $u_{1}, \ldots, u_{n_{u}}$ and average WFS slopes $\alpha_{u, 1}, \alpha_{v, 1}, \ldots$, $\alpha_{u, n_{w}}, \alpha_{v, n_{w}}$ are collected to form the control input vector $u$ and the slope vector $s$. That is,

$$
u(t)=\left[\begin{array}{c}
u_{1}(t) \\
\vdots \\
u_{n_{u}}(t)
\end{array}\right], \quad s(t)=\left[\begin{array}{c}
\alpha_{u, 1} \\
\alpha_{v, 1} \\
\vdots \\
\alpha_{u, n_{w}} \\
\alpha_{v, n_{w}}
\end{array}\right] .
$$

Equations (3.1), (3.2) are translated into modal coordinates using vector notation as

$$
w_{r}(t)=w_{a}(t)-w_{m}(t),
$$

and

$$
w_{m}(t)=M_{m} u(t),
$$


where $M_{m}$ is called the mirror influence matrix. The slope signal $s$ is expressed as

$$
s(t)=M_{w} w_{r}(t),
$$

where we denote $M_{w}$ the WFS matrix. As mentioned by Looze (2005), the output of the CCD detector, intrinsically a discrete-time signal, integrates over the sampling period $T$ the delayed slope

$$
\tilde{s}(t)=s(t-\tau) .
$$

We call $\tau$ the continuous time measurement delay which is the sum of the CCD's readout time and of the slopes' computation time. Thus, the output of the CCD based sensor is

$$
y(t)=\frac{1}{T} \int_{t-T}^{t} \tilde{s}(\sigma) d \sigma+n(t),
$$

where $n(t)$ is an additive noise caused by the photon fluctuations and by the detector's readout noise.

\subsection{Disturbance rejection MIMO feedback loop}

If we refer to the feedback block diagram depicted in Figure 13, Equations (3.5), (3.6), (3.7), (3.8), and (3.9) define the continuous time part of the sampled-data feedback system shown in Figure 13. The regulated output is $z(t)=w_{r}(t)$, the disturbance input is $d(t)=w_{a}(t)$, and the actuator output is $v(t)=w_{m}(t)$. The actuator/DM transfer function is simply

$$
G_{1}(p)=M_{m}
$$

In Figure 13, the sensor/WFS is described by the transfer function

$$
G_{2}(p)=\left(e^{-\tau p} I\right)\left(\frac{1-e^{-T p}}{T p} I\right) M_{w}
$$

As proposed in the paper (Demerle et al. 1994), a first approach, the emulation design method presented in Section 2.4.2, approximates the AO feedback system with the continuous time feedback system shown in Figure 7. In Section 2.4.2 we have considered a single-input single-output (SISO) feedback system but here the feedback loop signals may have large dimensions: this is the multiple-input and multiple-output (MIMO) feedback system depicted in Figure 25.

The Equation (2.6) established for a single-input single-output (SISO) system becomes

$$
\begin{aligned}
\mathcal{L}\left\{w_{r}\right\}(p)= & \overbrace{\left(I+G_{1}(p) K(p) G_{2}(p)\right)^{-1}}^{S(p)} \mathcal{L}\left\{w_{a}\right\}(p) \\
& -\underbrace{\left(I+G_{1}(p) K(p) G_{2}(p)\right)^{-1} G_{1}(p) K(p)}_{T(p)} \mathcal{L}\{n\}(p) .
\end{aligned}
$$




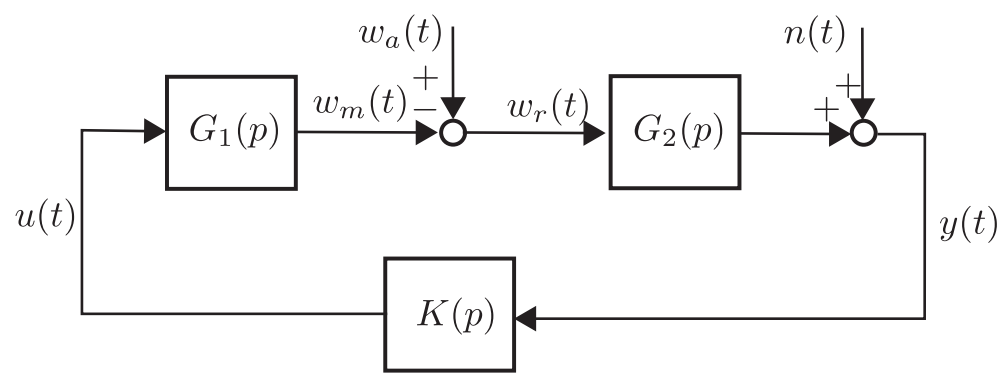

Fig. 25. An approximation of an AO MIMO feedback system.

where the following terminology remains:

(i) $L(p)=G_{1}(p) K(p) G_{2}(p)$ is the loop transfer function;

(ii) $S(p)=(I+L(p))^{-1}$ is the sensitivity transfer function;

(iii) $T(p)=S(p) G_{1}(p) K(p)$ is the noise sensitivity transfer function.

The disturbance rejection performance is entirely determined by transfer functions $S$ and $T$. At this step no assumption is made for the type of controller (optimized modal controller, linear quadratic Gaussian control, ...) for the set of the perturbation inputs $w_{a}$ and $n$. The performance criterion, the "size" of the residual wavefront $w_{r}$ is not defined either. A possible approach sketched in Section 2.2, involves the frequency response analysis generalized for MIMO systems which provides some crucial information about the system performances (stability, disturbance rejection, command input peak value), see for instance the book 2007. Another way is to evaluate the "size" of the residual wavefront $w_{r}$ in terms of the variance (mean-square error) $\mathbf{E}\left[w_{r}(k)^{T} w_{r}(k)\right]$ when stochastic signals $w_{a}, n_{w}$ are considered zero mean, stationary and independent. The Maréchal approximation (Born \& Wolf 1999) can be invoked to show that bounding the mean-square error of the residual wavefront ensures satisfactory imaging performance of AO systems. Thus, in the frequency domain, the variance can be written as

$$
\begin{aligned}
& \mathbf{E}\left[\left\|w_{r}(t)\right\|^{2}\right]=\frac{1}{2 \pi} \int_{-\infty}^{\infty} \operatorname{Tr}\left(S(j \omega) \Phi_{w_{a}}(j \omega T) S(-j \omega)^{T}\right) d \omega \\
& \quad+\frac{1}{2 \pi} \int_{-\infty}^{\infty} \operatorname{Tr}\left(T(j \omega) \Phi_{n}(j \omega) T(-j \omega)^{T}\right) d \omega
\end{aligned}
$$

where $\Phi_{w_{a}}$ and $\Phi_{n}$ are the power spectral densities of the input signals $w_{a}$ and $n_{w}$. The first term of the right hand side of Equation (3.11) represents the contribution of the atmospheric wavefront and the latter the contribution of the WFS measurement noise. The optimized modal gain integral control (OMGI) proposed by Gendron \& Léna (1994) and its improvements, see (Dessenne et al. 1998), is a well-established method to tackle this control problem. 


\subsection{Optimized modal gain integral control}

The key idea of the approach is to reconstruct the wavefront using the WFS measurement $y$ and to consider the linear relation (3.7). The WFS matrix $M_{w}$ can be expressed into its singular value decomposition, see (Laub 2004)

$$
M_{w}=U \Sigma V^{T}
$$

where $U, V$ are orthogonal matrices. We assume that rank $\left(M_{w}\right)=n_{b}$ and

$$
U=\left[\begin{array}{ll}
U_{1} & U_{2}
\end{array}\right], \quad \Sigma=\left[\begin{array}{l}
S \\
0
\end{array}\right], \text { with } S=\operatorname{diag}\left(\sigma_{i}\right),
$$

where terms $\sigma_{i}$ are positive singular values of the matrix $M_{w}$. We define $M_{w}^{\dagger}=$ $V S^{-1} U_{1}^{T}$ as the Moore-Penrose pseudoinverse of the matrix $M_{w}$, see (Laub 2004). We also denote rank $\left(M_{m}\right)=n_{b}$ and we call $M_{m}^{\dagger}$ the Moore-Penrose pseudoinverse of the matrix $M_{m}$. An integral (modal) controller can be defined as

$$
K(p)=M_{m}^{\dagger}\left(\frac{1}{p} K_{I}\right) M_{w}^{\dagger}
$$

where $K_{I}$ is the matrix integrator gain to design. We consider a new atmospheric wavefront signal $\tilde{w}_{a}$, and a new sensor noise signal $\tilde{n}$, such that

$$
w_{a}=V \tilde{w}_{a}, \quad n=U_{1} S \tilde{n},
$$

and a new residual wavefront signal

$$
\tilde{w}_{r}=V^{T} w_{r}
$$

The block diagram of the feedback system is depicted in Figure 26.

Despite the complexity of the block diagram, a change of signals allows us to obtain a straightforward expression of the residual wavefront

$$
\begin{aligned}
\mathcal{L}\left\{\tilde{w}_{r}\right\}= & \underbrace{\left(I+\frac{1}{p} \tilde{K}_{I} e^{-\tau p} \frac{1-e^{-T p}}{T p}\right)^{-1}}_{\tilde{S}} \mathcal{L}\left\{\tilde{w}_{a}\right\} \\
& -\underbrace{\left(I+\frac{1}{p} \tilde{K}_{I} e^{-\tau p} \frac{1-e^{-T p}}{T p}\right)^{-1} \frac{1}{p} \tilde{K}_{I}}_{\tilde{T}} \mathcal{L}\{\tilde{n}\}
\end{aligned}
$$

where the matrix gain is $\tilde{K}_{I}=V^{T} K_{I} V$. If we fix the matrix gain such that $\tilde{K}_{I}=\operatorname{diag}\left(\tilde{k}_{i}\right)$, then the MIMO transfer functions $\tilde{S}$ and $\tilde{T}$ are diagonal: the MIMO control problem reduces to $n_{b}$ independent SISO control problems. We call $\tilde{S}_{i}\left(\tilde{T}_{i}\right)$ the ith diagonal entry of the sensitivity transfer function $S$ (the noise 


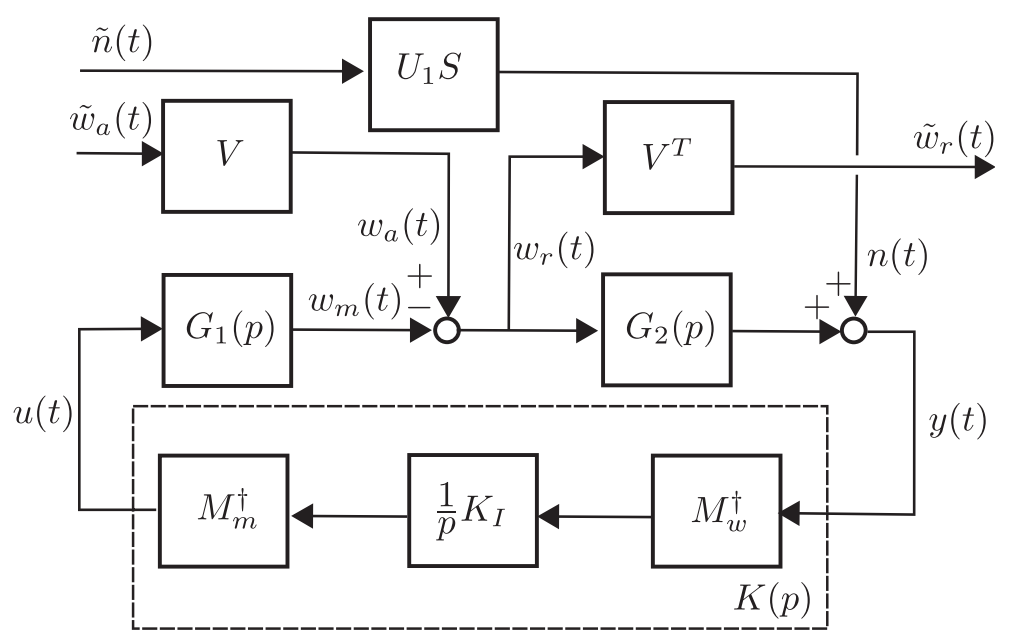

Fig. 26. Modal feedback system.

sensitivity transfer function $T$ ). Thus, the variance of each component can be written as

$$
\mathbf{E}\left[\left\|\tilde{w}_{r, i}(t)\right\|^{2}\right]=\frac{1}{2 \pi} \int_{-\infty}^{\infty}\left|\tilde{S}_{i}(j \omega)\right|^{2} \Phi_{\tilde{w}_{a, i}}(j \omega) d \omega+\frac{1}{2 \pi} \int_{-\infty}^{\infty}\left|\tilde{T}_{i}(j \omega)\right|^{2} \Phi_{\tilde{n}_{i}}(j \omega) d \omega,
$$

where $\Phi_{\tilde{w}_{a, i}}(j \omega)$ and $\Phi_{\tilde{n}_{i}}(j \omega)$ are the power spectral densities of the ith component of vector signals $\tilde{w}_{a}$ and $\tilde{n}$. The integral gain $\tilde{k}_{i}$ is tuned using the loop shaping approach sketched in Section 2.3.2 to minimize the variance of the ith component which induces the minimization of the variance $\mathbf{E}\left[w_{r}(t)^{T} w_{r}(t)\right]$. Then, the controller matrix gain is computed as

$$
K_{I}=V \tilde{K}_{I} V^{T}
$$

The main advantage of the optimized modal gain integral control, which explains its success in practice, is to express some of the controller's signals in the modal base which facilitates the physical interpretation. Furthermore it is intrinsically a frequency approach: the analysis of the AO feedback system's performance is straightforward. The well established OMGI control offers interesting abilities. Constant additive disturbances as actuator offset are intrinsically rejected. The real time computational cost is reasonable and induces limited delay. The method can be used when the knowledge of the disturbance temporal dynamics is weak. Some shortcomings have been mentioned in the literature. The integral controller can be transformed into an observer based controller structure, see (Kulcsár et al. 2006). The observer is not stable and the control $u$ may blow up. On a simplified SCAO configuration some authors Conan et al. (2011) indicated that more advanced control approaches such as linear quadratic Gaussian control ensure better performances. 


\section{Modern feedback control: LQG method for adaptive optics}

\subsection{Towards linear quadratic Gaussian control}

\subsubsection{Adaptive optics feedback loop}

The WFS Equations (3.7), (3.8), and (3.9) provide a linear relationship between the temporal average of the residual wavefront over the sampling period $T$ and the discrete time measurement (2.16) corrupted by a measurement noise. Thus, we can write the discrete time residual wavefront $w_{r}(k)$ as the average of the continuous time residual wavefront $w_{r}(t)$

$$
w_{r}(k)=\frac{1}{T} \int_{(k-1) T}^{k T} w_{r}(t) d t .
$$

The same temporal discretization (4.1) is done for the mirror wavefront $w_{m}(k)$ and the atmospheric wavefront $w_{a}(k)$. The WFS Equations (3.7), (3.8), (3.9), and (2.16) are transformed into difference equation. We obtain in the frequency domain

$$
\mathcal{Z}\{y\}=\underbrace{z^{-k_{y}} M_{w}}_{G_{1}} \mathcal{Z}\left\{w_{r}\right\}+\mathcal{Z}\{n\},
$$

where $n$ is an additive measurement noise and where $k_{y}$ is the measurement delay such that $\tau=k_{y} T$. Equations (3.5) and (3.6) become

$$
\mathcal{Z}\left\{w_{r}\right\}=\mathcal{Z}\left\{w_{a}\right\}-\underbrace{M_{w} z^{-k_{u}}}_{G_{2}} \mathcal{Z}\{u\},
$$

where $k_{u} \geq 1$ represents the control input delay. We call $G_{1}(z)$ the DM transfer function and $G_{2}(z)$ the WFS transfer function. The block diagram of the discrete time AO feedback system is shown in Figure 21. Here the AO loop is a MIMO feedback system. The regulated output response (2.25) established for a SISO system becomes

$$
\begin{aligned}
\mathcal{Z}\left\{w_{r}\right\}(z)= & \overbrace{\left(I+G_{1}(z) K(z) G_{2}(z)\right)^{-1}}^{S(z)} \mathcal{Z}\left\{w_{a}\right\}(z) \\
& -\underbrace{\left(I+G_{1}(z) K(z) G_{2}(z)\right)^{-1} G_{1}(z) K(z)}_{T(z)} \mathcal{Z}\{n\}(z) .
\end{aligned}
$$

where the following terminology remains:

(i) $L(z)=G_{1}(z) K(z) G_{2}(z)$ is the loop transfer function;

(ii) $S(z)=(I+L(z))^{-1}$ is the sensitivity transfer function;

(iii) $T(z)=S(z) G_{1}(z) K(z)$ is the noise sensitivity transfer function. 
Up to now the framework is identical to the approach presented for the continuous time feedback loop in the frequency domain. However we have to keep in mind that here we adopt the point of view of the digital computer and that the regulated output $w_{r}(k)$ is the temporal average of the "real" regulated output $w_{r}(t)$. This approach is relevant when the choice of the sampling period $T$ is not critical in regards with the dynamics of the atmospheric wavefront. We assume that signals $w_{a}, n_{w}$ are zero mean, stationary and independent stochastic signals. Thus, in the frequency domain, the variance $\mathbf{E}\left[\left\|w_{r}(k)\right\|^{2}\right]$ can be written as

$$
\begin{aligned}
\mathbf{E}\left[\left\|w_{r}(k)\right\|^{2}\right]= & \frac{T}{2 \pi} \int_{0}^{\frac{2 \pi}{T}} \operatorname{Tr}\left(S\left(e^{j \omega T}\right) \Phi_{w_{a}}(\omega) S\left(e^{-j \omega T}\right)^{T}\right) d \omega \\
& +\frac{T}{2 \pi} \int_{0}^{\frac{2 \pi}{T}} \operatorname{Tr}\left(T\left(e^{j \omega T}\right) \Phi_{n}(\omega) T\left(e^{-j \omega T}\right)^{T}\right) d \omega
\end{aligned}
$$

where $\Phi_{w_{a}}$ and $\Phi_{n}$ are the power spectral densities of the input signals $w_{a}$ and $n$. The first term of the right hand side of Equation (4.5) represents the contribution of the atmospheric wavefront and the latter the contribution of the WFS measurement noise. Equation (4.5) indicates the frequency range where the frequency responses $S\left(e^{j \omega T}\right)$ and $T\left(e^{j \omega T}\right)$ have to be small. Power spectral densities $\Phi_{w_{a}}$ and $\Phi_{n}$ can be seen as weighting functions for performance objective (4.5). The control problem can be formulated as finding the control law that minimizes the variance $\mathbf{E}\left[\left\|w_{r}(k)\right\|^{2}\right]$ To take into account more accurately the information of the atmospheric wavefront we have to build a model of the temporal evolution of $w_{a}(k)$.

\subsubsection{Identified atmospheric wavefront model}

The power spectral densities $\Phi_{w_{a}}$ may be factored as

$$
\Phi_{w_{a}}(w)=G_{a}\left(e^{j \omega T}\right) G_{a}\left(e^{-j \omega T}\right)^{T}
$$

and the atmospheric wavefront $w_{a}$ is assumed to be the output of a causal and stable diagonal transfer function matrice $G_{a}$ driven by a white noise $n_{a}$ having a unitary covariance matrix. To take into account the oscillating behavior of $w_{a}(k)$ a second order diagonal AR model is considered

$$
A_{0} w_{a}(k)+A_{1} w_{a}(k-1)+A_{2} w_{a}(k-2)=n_{a}(k),
$$

where diagonal matrices $\left(A_{0}, A_{1}, A_{2}\right)$ are the AR parameters. The computation of the parameters is carried out with the Burg algorithm, see (Burg 1975), which minimizes the sum of the squares of the forward and backward prediction errors. In the frequency domain we obtain

$$
\mathcal{Z}\left\{w_{a}\right\}=\underbrace{\left(A_{0} z^{2}+A_{1} z+A_{2}\right)^{-1} z^{2}}_{G_{a}} \mathcal{Z}\left\{n_{a}\right\} .
$$

which defines the atmospheric wavefront filter $G_{a}$. The AO block diagram is depicted in Figure 27 where the different loop signals are mentioned. 


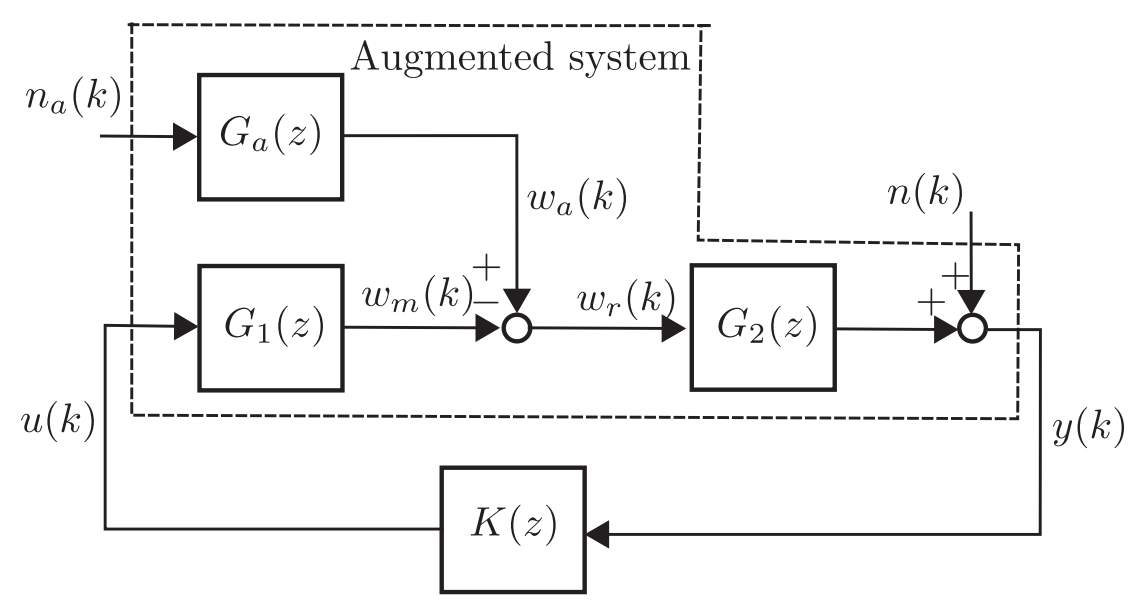

Fig. 27. AO discrete-time system block-diagram including the atmospheric model.

\subsubsection{Performance objective in the time domain}

In the time domain, the AO control problem can be formulated as finding the control law that minimizes the empirical variance of the residual wavefront, averaged over a large exposure time $T_{e}$

$$
\mathbf{E}\left[\left\|w_{r}(t)\right\|^{2}\right]=\lim _{T_{e} \rightarrow \infty} \frac{1}{T_{e}} \int_{0}^{T_{e}}\left\|w_{r}(t)\right\|^{2} d t,
$$

which is the time domain counterpart of (3.11) for a stationary ergodic process and the "true" imaging performance index. Several authors Kulcsár et al. (2006), Looze (2007) demonstrated that the minimization of the residual wavefront variance $\mathbf{E}\left[\left\|w_{r}(t)\right\|^{2}\right]$ can be performed using the discrete-time model of the hybrid AO system without loss of optimality. Therefore, the performance objective to minimize, in the discrete-time domain is translated as

$$
\mathbf{E}\left[\left\|w_{r}(k)\right\|^{2}\right]=\lim _{N \rightarrow \infty} \frac{1}{N} \sum_{k=1}^{N}\left\|w_{r}(k)\right\|^{2},
$$

which is the time domain counterpart of (4.5). This last control objective can be minimized using LQG design approach using a state-space description of the augmented plant (DM, WFS, atmospheric wavefront model) as discussed in Section 4.3.

\subsection{LQG control framework}

\subsubsection{State space equation}

The state space method is based on the description of system equation in terms of $n$ first-order difference equations, which may be combined into a first-order 
vector-matrix difference equation. The state space equation of a discrete time system can be written

$$
\begin{aligned}
x(k+1) & =A x(k)+B e(k) \\
s(k) & =C x(k)+D u(k) .
\end{aligned}
$$

Here $x \in \mathbf{R}^{n}$ is the state of the system, $e \in \mathbf{R}^{m}$ is the input, and $s \in \mathbf{R}^{r}$ is the output. For example consider the AR difference Equation (4.6) when the signals $w_{a}$ and $n_{a}$ are scalars

$$
a_{0} w_{a}(k+1)+a_{1} w_{a}(k)+a_{2} w_{a}(k-1)=n_{a}(k),
$$

where real scalars $a_{0}, a_{1}, a_{2}$ are given. To convert this equation into the state space Equation (4.9), we define $x_{1}(k)=w_{a}(k), x_{2}(k)=w_{a}(k-1), e(k)=n_{a}(k)$, and $s(k)=w_{a}(k)$. The first-order difference equations are then

$$
\begin{aligned}
x_{1}(k+1) & =w_{a}(k+1)=-\frac{a_{1}}{a_{0}} w_{a}(k)-\frac{a_{2}}{a_{0}} w_{a}(k-1)+\frac{1}{a_{0}} n_{a}(k) \\
& =-\frac{a_{1}}{a_{0}} x_{1}(k)-\frac{a_{2}}{a_{0}} x_{2}(k)+\frac{1}{a_{0}} e(k) \\
x_{2}(k+1) & =w_{a}(k)=x_{1}(k) \\
s(k) & =w_{a}(k)=x_{1}(k) .
\end{aligned}
$$

We can write this in matrix/vector form as

$$
\begin{aligned}
{\left[\begin{array}{l}
x_{1}(k+1) \\
x_{2}(k+1)
\end{array}\right] } & =\left[\begin{array}{rr}
-\frac{a_{1}}{a_{0}} & -\frac{a_{2}}{a_{0}} \\
1 & 0
\end{array}\right]\left[\begin{array}{l}
x_{1}(k) \\
x_{2}(k)
\end{array}\right]+\left[\begin{array}{c}
\frac{1}{a_{0}} \\
0
\end{array}\right] e(k) \\
s(k) & =\left[\begin{array}{ll}
1 & 0
\end{array}\right]\left[\begin{array}{l}
x_{1}(k) \\
x_{2}(k)
\end{array}\right]+0 e(k) .
\end{aligned}
$$

If we pose

$$
A=\left[\begin{array}{rr}
-\frac{a_{1}}{a_{0}} & -\frac{a_{2}}{a_{0}} \\
1 & 0
\end{array}\right], \quad B=\left[\begin{array}{c}
\frac{1}{a_{0}} \\
0
\end{array}\right], \quad C=\left[\begin{array}{ll}
1 & 0
\end{array}\right], \quad D=0,
$$

we obtain the state space Equation (4.9). In the general case, the atmospheric wavefront model $G_{a}$ can be written in state space form as

$$
\begin{aligned}
x_{a}(k+1) & =A_{a} x_{a}(k)+B_{a} n_{a}(k), \\
y_{a}(k) & =C_{a} x_{a}(k),
\end{aligned}
$$

where the state $x_{a} \in \mathbf{R}^{2 n_{b}}$ is $x_{a}(k)=\left[w_{a}(k)^{T} \quad w_{a}(k-1)^{T}\right]^{T}$ and where state space matrices are

$$
A_{a}=\left[\begin{array}{cc}
-A_{0}^{-1} A_{1} & -A_{0}^{-1} A_{2} \\
I & 0
\end{array}\right], \quad B_{a}=\left[\begin{array}{c}
A_{0}^{-1} \\
0
\end{array}\right], \quad C_{a}=\left[\begin{array}{ll}
I & 0
\end{array}\right] .
$$




\subsubsection{Linear quadratic Gaussian control}

The discrete-time LQG control theory considers that the system is linear and that the disturbance (plant noise) and the measurement noise inputs are stochastic. Thus, the system is described by the state-space representation

$$
\begin{aligned}
x(k+1) & =A x(k)+B u(k)+w(k) \\
y(k) & =C x(k)+v(k),
\end{aligned}
$$

where $x \in \mathbf{R}^{n}$ is the state vector, $u \in \mathbf{R}^{n_{u}}$ the command input, $y \in \mathbf{R}^{n_{y}}$ the measured output, and where $w \in \mathbf{R}^{n}$ represents the disturbance input and $v \in$ $\mathbf{R}^{n_{y}}$ is the measurement noise input. We assume that Gaussian noise processes $w(k)$ and $v(k)$ are mutually independent, zero mean white noises with covariance $\mathbf{E}\left[w(k) w^{T}(l)\right]=W \delta(k-l)$ and $\mathbf{E}\left[v(k) v^{T}(l)\right]=V \delta(k-l)$, respectively. It is supposed that the pair $(A, B),\left(A, W^{1 / 2}\right)$ are stabilizable and the pair $(A, C)$ is detectable.

The LQG control problem is to find the optimal control $u(k)$ for system $(4.12)$ that minimizes the infinite horizon quadratic cost criterion

$$
J=\lim _{N \rightarrow \infty} \frac{1}{N} \mathbf{E}\left[\sum_{k=0}^{N-1} x(k)^{T} Q x(k)+u(k)^{T} R u(k)\right],
$$

with given weighting matrices $Q=Q^{T} \geq 0, R=R^{T}>0$ and the pair $\left(A, Q^{1 / 2}\right)$ detectable.

The solution of the LQG control problem is then provided by the interconnection of a linear quadratic regulator and a state estimator. This result is known in linear optimal control theory as the Separation Principle, see (Kwakernaak \& Sivan 1972; Anderson \& Moore 1990). The optimal control sequence minimizing the cost function (4.13) is given by the state-feedback control law

$$
u(k)=-K \hat{x}(k),
$$

where $\hat{x}$ is the optimal estimate of the state $x$. The state-feedback gain $K$ is a constant matrix

$$
K=\left(R+B^{T} P B\right)^{-1} B^{T} P A,
$$

where the matrix $P=P^{T}$ is the unique positive-semidefinite solution of the control discrete-time algebraic Riccati equation (DARE)

$$
P=A^{T} P A-A^{T} P B\left(B^{T} P B+R\right)^{-1} B^{T} P A+Q
$$

Note that the conditions $R>0,(A, B)$ detectable and $\left(A, Q^{1 / 2}\right)$ detectable can be relaxed, see (Bitmead \& Gevers 1991; Dorato \& Levis 1971). The optimal state estimation which minimizes the variance of the estimation error $\mathbf{E}\left[\mid \hat{x}(k)-x(k) \|^{2}\right]$, is performed through a standard Kalman predictor filter with

$$
\hat{x}(k+1)=A \hat{x}(k)+B u(k)+L(y(k)-C \hat{x}(k)),
$$


where $L$ is the observer gain

$$
L=A X C^{T}\left(C X C^{T}+B\right)^{-1} .
$$

where the matrix $X=X^{T}$ is the unique positive-semidefinite solution of the estimation DARE

$$
X=A X A^{T}-A X C^{T}\left(C X C^{T}+V\right)^{-1} C X A^{T}+W .
$$

\subsection{Application of $L Q G$ control to the adaptive optics system}

\subsubsection{AO state space system}

In the sequel we consider a unitary input delay $k_{u}=1$ and a unitary output delay $k_{y}=1$. The "augmented system", depicted in Figure 27, is described by the state space Equation (4.9) where the signals are defined as follows.

1. The state vector $x$ is split in two parts $x=\left[\begin{array}{ll}x_{m}^{T} & x_{a}^{T}\end{array}\right]^{T}$. The state $x_{m}(k)=$ $\left[w_{m}(k)^{T} w_{m}(k-1)^{T}\right]^{T}$ represents the plant dynamics (DM \& WFS) and state $x_{a}(k)$ corresponds to the perturbation dynamics (4.10).

2. The state noise is $w=\left[\begin{array}{c}0 \\ B_{a}\end{array}\right] n_{a}$ and the measurement noise is $v=n$.

The state space matrices of the augmented system (DM, WFS, ATM) are defined as

$$
A=\left[\begin{array}{cc}
A_{m} & 0 \\
0 & A_{a}
\end{array}\right], \quad B=\left[\begin{array}{c}
B_{m} \\
0
\end{array}\right], \quad C=M_{w}\left[\begin{array}{ll}
C_{m} & C_{a}
\end{array}\right] .
$$

The state space matrices of the plant are

$$
A_{m}=\left[\begin{array}{ll}
0 & 0 \\
I & 0
\end{array}\right], \quad B_{m}=\left[\begin{array}{c}
M_{m} \\
0
\end{array}\right], \quad C_{m}=\left[\begin{array}{ll}
0 & -I
\end{array}\right],
$$

and state-space matrices $\left(A_{a}, B_{a}, C_{a}\right)$ are given in (4.11).

The special form of state space matrices (4.20) can be exploited to simplify the resolution of the Riccati equations, see (Bitmead et al. 1990). For the presentation of the following results, matrices $P, X$ and $Q$ are partitioned conformally with the matrix $A$, that is

$$
P=\left[\begin{array}{cc}
P_{m} & P_{0} \\
P_{0}^{T} & P_{a}
\end{array}\right], \quad X=\left[\begin{array}{cc}
X_{m} & X_{0} \\
X_{0}^{T} & X_{a}
\end{array}\right], \quad Q=\left[\begin{array}{ll}
Q_{m} & Q_{0} \\
Q_{0}^{T} & Q_{a}
\end{array}\right]
$$

\subsubsection{Solving the control DARE}

The control DARE (4.16) can be simplified to obtain solutions for the individual blocks of $P$. We have to find the matrix $P_{m}=P_{m}^{T}$ the unique positive-semidefinite solution of the reduced order DARE

$$
P_{m}=A_{m}^{T} P_{m} A_{m}-A_{m}^{T} P_{m} B_{m}\left(B_{m}^{T} P_{m} B_{m}+R\right)^{-1} B_{m}^{T} P_{m} A_{m}+Q_{m}
$$


The state-feedback gain (4.15) becomes $K=\left[\begin{array}{ll}K_{m} & K_{a}\end{array}\right]$ with

$$
K_{m}=\left(B_{m}^{T} P_{m} B_{m}+R\right)^{-1} B_{m}^{T} P_{m} A_{m}
$$

We search matrix $P_{0}$ which is a solution of the following discrete-time Sylvester equation

$$
P_{0}=\left(A_{m}-B_{m} K_{m}\right)^{T} P_{0} A_{a}+Q_{0}
$$

We obtain

$$
K_{a}=\left(B_{m}^{T} P_{m} B_{m}+R\right)^{-1} B_{m}^{T} P_{0} A_{a} .
$$

The special form of state space matrices (4.21) imply that $K_{m}=0$ and that

$$
K_{a}=-\left(R+M_{m}^{T} M_{m}\right)^{-1} M_{m}^{T} C_{a} A_{a}^{2}
$$

\subsubsection{Solving the estimation DARE}

The estimation error can be written as $\tilde{x}^{T}=\left[\begin{array}{ll}\tilde{x}_{m}^{T} & \tilde{x}_{a}^{T}\end{array}\right]=\left[\begin{array}{ll}\hat{x}_{m}^{T}-x_{m}^{T} & \hat{x}_{a}^{T}-x_{a}^{T}\end{array}\right]$. The state $x_{m}$ is a deterministic signal and thus $\tilde{x}_{m}=0$ which simplifies the blocks $X_{m}=0, X_{0}=0$. The estimation DARE (4.19) can be simplified to obtain solutions for the individual blocks of $X$. Thus the matrix $X_{a}=X_{a}^{T}$ is the unique positive-semidefinite solution of the reduced order DARE

$$
X_{a}=A_{a} X_{a} A_{a}^{T}-A_{a} X_{a} C_{a}^{T}\left(C_{a} X_{a} C_{a}^{T}+V\right)^{-1} C_{a} X_{a} A_{a}^{T}+B_{a} B_{a}^{T} .
$$

The observer gain (4.18) becomes $L=\left[\begin{array}{c}0 \\ L_{a}\end{array}\right]$ with

$$
L_{a}=A_{a} X_{a} C_{a}^{T}\left(C_{a} X_{a} C_{a}^{T}+V\right)^{-1}
$$

\subsubsection{LQG controller}

The strictly proper, linear time invariant controller, is described by the state-space equation

$$
\begin{aligned}
\hat{x}(k+1) & =\hat{A} \hat{x}(k)+\hat{B} y(k) \\
u(k) & =\hat{C} \hat{x}(k)
\end{aligned}
$$

where the matrices $\hat{A}, \hat{B}, \hat{C}$ are

$$
\hat{A}=\left[\begin{array}{cc}
A_{m} & -B_{m} K_{a} \\
-L_{a} M_{w} C_{m} & A_{a}-L_{a} M_{w} C_{a}
\end{array}\right], \quad \hat{B}=\left[\begin{array}{c}
0 \\
L_{a}
\end{array}\right], \hat{C}=-\left[\begin{array}{ll}
0 & K_{a}
\end{array}\right] .
$$

Note that the LQG controller is equivalently described by the discrete time transfer function

$$
K(z)=\hat{C}(z I-\hat{A})^{-1} \hat{B}
$$

which is a convenient form (i) to analyze the AO feedback system depicted in Figure 27; (ii) to interpret the AO performance index (4.5). 


\subsection{LQG controller design}

We consider an 8-m telescope without obstruction and the $512 \times 512$-pixels wavefronts projected over 44 Zernike $\left(n_{b}=44\right)$. The physical modeling has been performed by means of the Software Package CAOS (Carbillet et al. 2005), developed within the CAOS problem-solving environment (PSE), see (Carbillet et al. 2010). The computation of the LQG state-space matrices (4.20) is carried out using Matlab software and the Control system toolbox and involves the following steps.

Step 1: AO discrete-time state-space computation. DM controls perfectly low spatial frequencies with $n_{u}=44$ actuators and consequently $M_{m}=I_{n_{b}}$. The WFS device is a $8 \times 8\left(\Rightarrow n_{y}=52\right)$ subaperture Shack-Hartmann WFS $\left(8 \times 80.2^{\prime \prime}\right.$ px/subap., $\lambda=700 \mathrm{~nm} \pm 150 \mathrm{~nm}, \Delta t=T=1 \mathrm{~ms}$.). The WFS influence matrix $M_{w}$ is determined from the WFS calibration simulation.

Using Software Package CAOS $500 \times 1 \mathrm{~ms}$ wavefronts propagated through an evolving 2-layers turbulent atmosphere $\left(r_{0}=10 \mathrm{~cm}\right.$ at $\lambda=500 \mathrm{~nm}, \mathcal{L}_{0}=25 \mathrm{~m}$, wind velocities $=8 \& 12 \mathrm{~m} / \mathrm{s}$ ) are obtained. After the projection on the Zernike base, the signal $w_{a}$ is modeled as the output of an AR system using the approach presented in Section 4.1.2. The computation of the parameters is carried out with the Burg algorithm, see (Burg 1975), using the Signal Processing Toolbox of Matab and permits to obtain state space matrices (4.11). Then, the computation of the LQG state space matrices is obvious using Equation (4.20).

Step 2: Additive noise covariance estimation. Covariance matrix $V$ for LQG design is a tuning parameter which dictates the performance of the AO control loop. We use the empirical covariance matrix obtained from a photon noise calibration from our CAOS simulations. Note that this needs anyway to be refined for future developments.

Step 3: controller design. To minimize the performance objective $\mathbf{E}\left[\left\|w_{r}(k)\right\|^{2}\right]$ given in the discrete-time domain (4.8) we consider the LQG performance index $J$ defined by (4.13) with the weigthing parameter $R=0$ (cheap control case). We have designed two kinds of optimal controller. LQG1 has been designed with the noise covariance matrix $V$ equal to zero, while for LQG2 we use the empirical covariance matrix built in step 2 .

\subsection{Discussion}

\subsubsection{A posteriori frequency analysis}

In the Figure 28-30 show the singular values of $S\left(e^{j \omega T}\right)$ in the left part, and the singular values of $T\left(e^{j \omega T}\right)$ in the right part. The maximum singular values are plotted in plain line, while the minimum singular values are plotted in dashed 

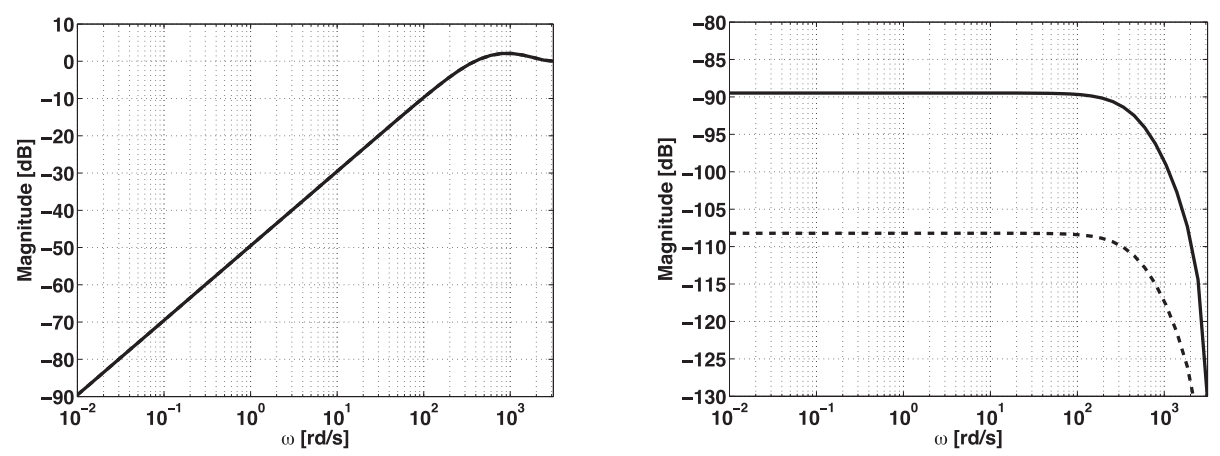

Fig. 28. Plot of singular values of $S\left(e^{j \omega T}\right)$ in the left part, and the singular values of $T\left(e^{j \omega T}\right)$ in the right part for integrator case (with a gain of 0.3 ).
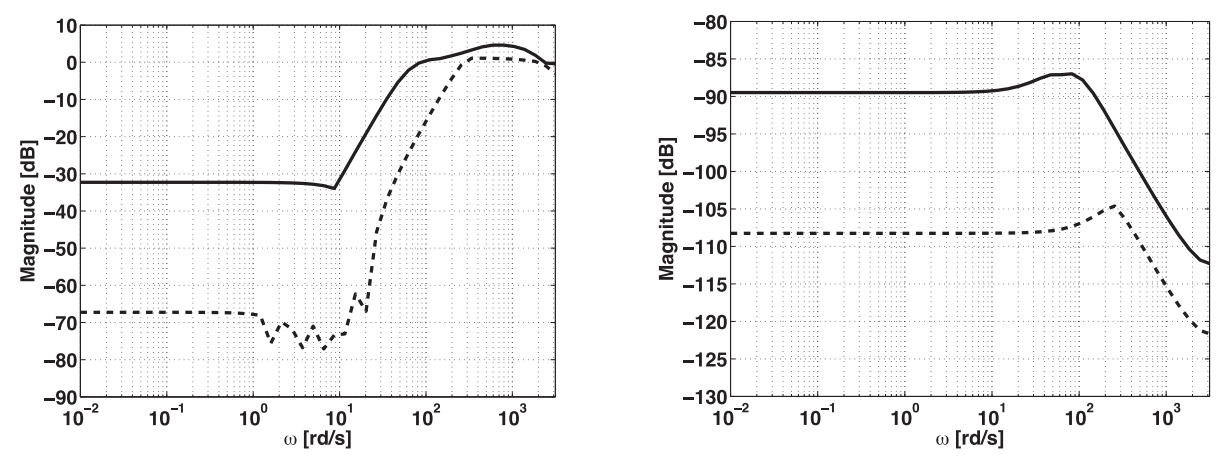

Fig. 29. Plot of singular values of $S\left(e^{j \omega T}\right)$ in the left part, and the singular values of $T\left(e^{j \omega T}\right)$ in the right part for LQG1 controller case.

line. The integrator case (with a gain of 0.3 ) is plotted in Figure 28, the LQG1 controller case in Figure 29, and the LQG2 controller case in Figure 30.

Note that the sensitivity transfer function $S$ for the LQG1 controller case shows that the LQG1 controller ensures a better rejection of the atmospheric wavefront than the LQG2 controller. If we check the frequency response of the noise rejection transfer function $T$, LQG1 design is more sensitive to noise than LQG2 design. The integrator case exhibits the worst frequency performance. These indications have to be confirmed by using CAOS end to end simulation.

\subsubsection{Performance comparison}

The time simulation has been performed by means of the Software Package CAOS. An ad hoc module, SSC, which stands for "Space-State Control", has been 

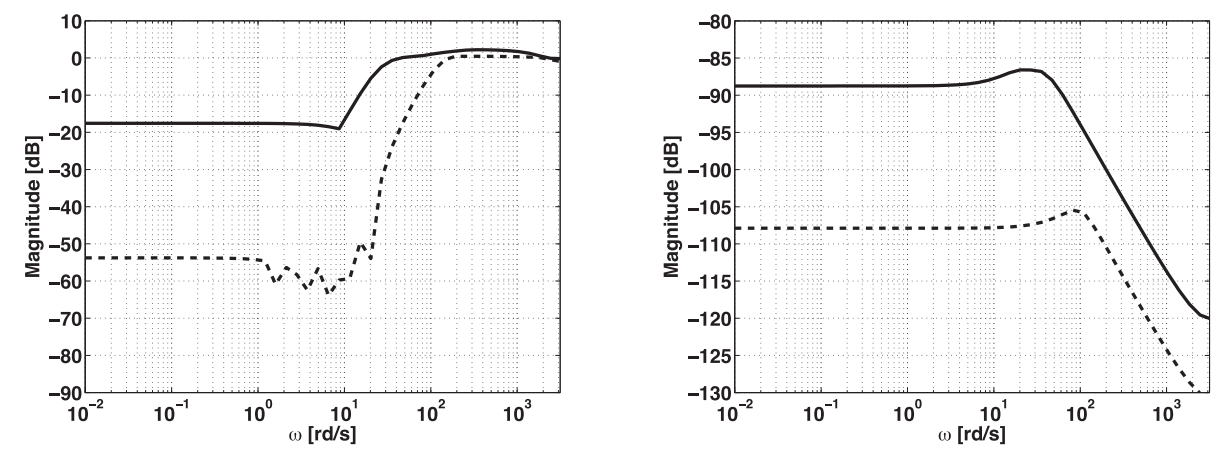

Fig. 30. Plot of singular values of $S\left(e^{j \omega T}\right)$ in the left part, and the singular values of $T\left(e^{j \omega T}\right)$ in the right part for LQG2 controller case.

developed especially for this study, also with the goal of making it publicly available with a future upgrade of the Software Package CAOS. Figure 31 shows the numerical modeling designed within the CAOS PSE.

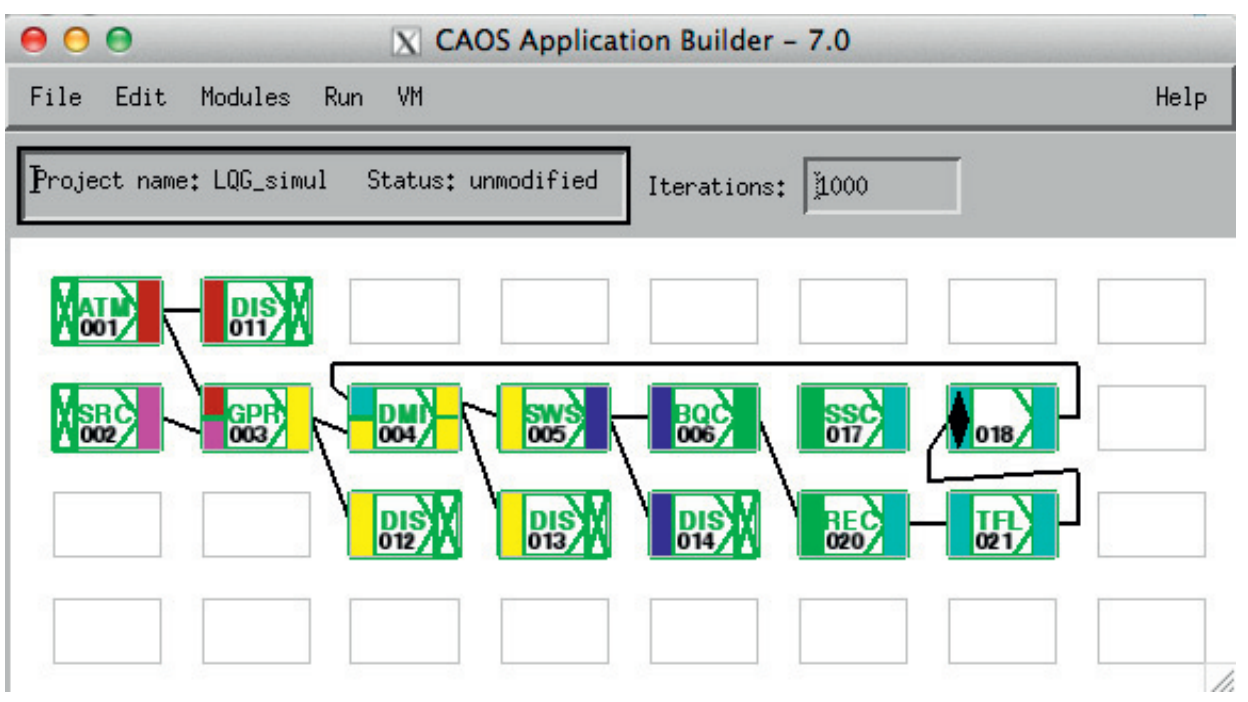

Fig. 31. CAOS numerical modeling of the AO system.

Figure 32 represents an example of running simulation. Left: the atmospherically-perturbed input wavefront. Middle: the corresponding Shack-Hartmann spots. Right: the resulting corrected wavefront.

For different operating conditions (star magnitude) we have obtained the following results sum up in Table 1. In bright conditions LGG and integral controllers 


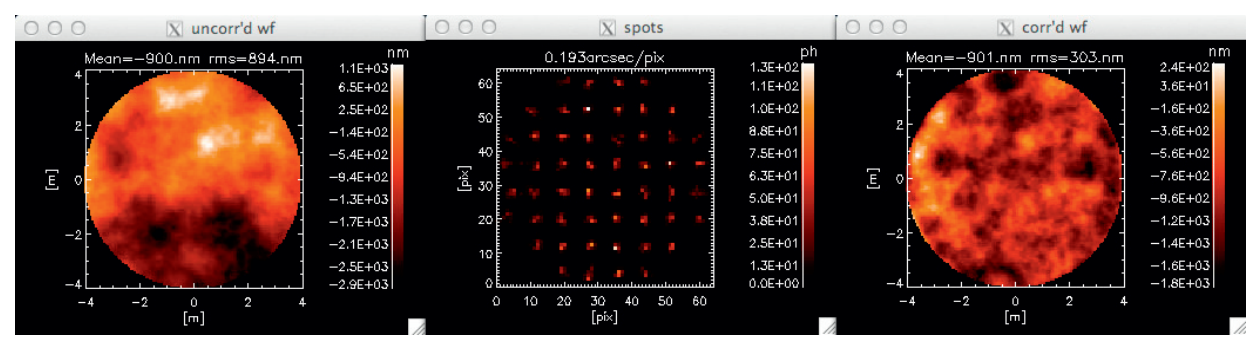

Fig. 32. CAOS running simulation.

Table 1. Obtained residual wavefront rms.

\begin{tabular}{|l|c|c|c|c|}
\hline & Photons/subaperture/T & Integrator & LQG1 & LQG2 \\
\hline no noise & $\infty$ & $\sim 268 \mathrm{~nm}$ & $\sim \mathbf{2 6 7} \mathbf{n m}$ & $\sim 271 \mathrm{~nm}$ \\
\hline mag 12 & $\sim 320$ & $\sim 269 \mathrm{~nm}$ & $\sim \mathbf{2 6 8} \mathbf{n m}$ & $\sim 271 \mathrm{~nm}$ \\
\hline mag 14 & $\sim 51$ & $\sim 272 \mathrm{~nm}$ & $\sim \mathbf{2 7 1} \mathbf{n m}$ & $\sim 273 \mathrm{~nm}$ \\
\hline mag 16 & $\sim 8.0$ & $\sim 296 \mathrm{~nm}$ & $\sim 297 \mathrm{~nm}$ & $\sim \mathbf{2 8 4} \mathbf{~ n m}$ \\
\hline mag 17 & $\sim 3.2$ & $\sim 350 \mathrm{~nm}$ & $\sim 356 \mathrm{~nm}$ & $\sim \mathbf{3 1 3} \mathbf{~ n m}$ \\
\hline mag 18 & $\sim 1.3$ & $\sim 471 \mathrm{~nm}$ & $\sim 475 \mathrm{~nm}$ & $\sim \mathbf{4 3 8} \mathbf{n m}$ \\
\hline
\end{tabular}

are equivalent until magnitude 14. In faint conditions (magnitude 16 to magnitude 18) the LQG2 controller induces better performance than the integral controller.

The authors are greatly indebted to the referee Céline Theys, for her helpful and constructive comments and Anthony Schutz for the computer assistance. The first author would like to thank Calypso Barnes for her valuable contribution to improve the quality of the english text.

\section{References}

Anderson, B., \& Moore, J.B., 1990, Optimal Control: Linear Quadratic Methods (Prentice-Hall)

Astrom, K.A., \& Wittenmark, B., 2011, Computer-Controlled Systems: Theory and Design (Dover Publications)

Bitmead, R.R., Gevers, M., \& Wertz, V., 1990, Adaptive Optimal Control: the Thinking Man's GPC (Prentice Hall Englewood Cliffs, NJ)

Bitmead, R.R., \& Gevers, M., 1991, Riccati Difference and Differential Equations: Convergence, monotonicity and stability, In The Riccati equation, ed. S. Bittanti, A.J. Laub \& J.C. Willems (Springer Verlag)

Boyd, S., 1993, Lecture Notes for E105, Introduction to Automatic Control (Stanford University)

Burg, J.P., 1975, Ph.D. Thesis, Maximum Entropy Spectral Analysis (Stanford University)

Born, M., \& Wolf, E., 1999, Principles of Optics: Electromagnetic Theory of Propagation, Interference and Diffraction of light (Cambridge University Press) 
Carbillet, M., Vérinaud, C., Femenía, B., Riccardi, A., \& Fini, L., 2005, MNRAS, 356, 1263

Carbillet, M., Desiderà, G., Augier, A., et al., 2010, Proc. SPIE, 7736, 773644

Conan, J.M., Raynaud, H.F., Kulcsár, C., Meimon, S., \& Sivo, G., 2011, Are Integral Controllers Adapted to the New Era of ELT Adaptive Optics? In AO4ELT2, Victoria, Canada, September

Dorato, P., \& Levis, A., 1971, Optimal Linear Regulators: the Discrete-time Case, IEEE Transactions on Automatic Control, 613

Dorf, R.C., \& Bishop, R.H., 1998, Modern Control Systems, Eight edition (AddisonWesley Longman Publishing Co., Inc., Boston, MA, USA)

Demerle, M., Madec, P.Y., \& Rousset, G., 1994, Servo-Loop Analysis for Adaptive Optics, In NATO Meeting, Cargèse, France, June 29-July 9, 1993, ONERA, TP, Vol. 423, 73

Dessenne, C., Madec, P.Y., \& Rousset, G., 1998, Appl. Opt., 37, 4623

Franklin, G.F., Powell, J.D., \& Emami-Naeni, A., 1991, Feedback Control of Dynamic Systems, Second edition (Addison-Wesley)

Franklin, G.F., Powell, J.D., \& Workman, M.L., 1990, Digital Control of Dynamic Systems, Second edition (Addison Wesley)

Gendron, E., \& Léna, P., 1994, A\&A (ISSN 0004-6361), 291

Kulcsár, C., Raynaud, H.F., Petit, C., Conan, J.M., \& de Lesegno, P.V., 2006, Appl. Opt, 39, 2525

Kwakernaak, H., \& Sivan, R., 1972, Linear Optimal Control Systems (John Wiley \& Sons)

Laub, A.J., 2004, Matrix Analysis for Scientists and Engineers, Society for Industrial Mathematics

Looze, D.P., 2005, Realization of Systems with CCD-based Measurements, Automatica, 41

Looze, D.P., 2006, J. Opt. Soc. Am., 23, 603

Looze, D.P., 2007, J. Opt. Soc. Am., 9, 2850

Noll, R.J., 1976, J. Opt. Soc. Am., 66, 207

Paschall, R.N., \& Anderson, D.J., 1993, Linear Quadratic Gaussian Control of a Deformable Mirror Adaptive Optics System with Time-delayed Measurements, Appl. Opt., 32

Paschall, R.N., Von Bokern, M.A., \& Welsh, B.M., 1991, Design of a Linear Quadratic Gaussian Controller for an Adaptive Optics System, In Proceedings of the 30th IEEE Conference on Decision and Control, 1761

Roddier, F., 1999, Adaptive Optics in Astronomy (Cambridge University Press)

Skogestad, S., \& Postlethwaite, I., 2007, Multivariable Feedback Control: Analysis and Design 